\title{
FLASH Early Science - Discovery of an intervening HI 21-cm absorber from an ASKAP survey of the GAMA 23 field
}

\author{
J. R. Allison, ${ }^{1,2 \star}$ E. M. Sadler, ${ }^{3,4,2}$ S. Bellstedt, ${ }^{5}$ L. J. M. Davies, ${ }^{5}$ S. P. Driver, ${ }^{5,6}$ \\ S. L. Ellison, ${ }^{7}$ M. Huynh, ${ }^{5,8}$ A. D. Kapińska, ${ }^{9}$ E. K. Mahony, ${ }^{4}$ V. A. Moss, ${ }^{4,3}$
} A. S. G. Robotham, ${ }^{5}$ M. T. Whiting, ${ }^{4}$ S. J. Curran, ${ }^{10}$ J. Darling ${ }^{11}$, A. W. Hotan, ${ }^{4}$ R. W. Hunstead,${ }^{3} \dagger$ B. S. Koribalski, ${ }^{4}$ C. D. P. Lagos,${ }^{2,5}$ M. Pettini, ${ }^{12}$ K. A. Pimbblet, ${ }^{13}$ and M. A. Voronkov ${ }^{4}$

${ }^{1}$ Sub-Dept. of Astrophysics, Department of Physics, University of Oxford, Denys Wilkinson Building, Keble Rd., Oxford, OX1 3RH, UK

${ }^{2}$ ARC Centre of Excellence for All-sky Astrophysics in 3 Dimensions (ASTRO 3D)

${ }^{3}$ Sydney Institute for Astronomy, School of Physics A28, University of Sydney, Sydney, NSW 2006, Australia

${ }^{4}$ CSIRO Astronomy and Space Science, PO Box 76, Epping, NSW 1710, Australia

${ }^{5}$ International Centre for Radio Astronomy Research (ICRAR), University of Western Australia, Crawley, WA 6009, Australia

${ }^{6}$ School of Physics \& Astronomy, University of St Andrews, North Haugh, St Andrews, KY16 9SS, UK,

${ }^{7}$ Department of Physics \& Astronomy, University of Victoria, Finnerty Road, Victoria, British Columbia, V8P 1A1, Canada

${ }^{8}$ CSIRO Astronomy and Space Science, 26 Dick Perry Avenue, Kensington WA 6151, Australia

${ }^{9}$ National Radio Astronomy Observatory, 1003 Lopezville Rd., Socorro NM 87801, USA

${ }^{10}$ School of Chemical and Physical Sciences, Victoria University of Wellington, PO Box 600, Wellington 6140, New Zealand

${ }^{11}$ Department of Astrophysical and Planetary Sciences, University of Colorado, 389 UCB, Boulder, CO 80309-0389, USA

${ }^{12}$ Institute of Astronomy, University of Cambridge, Madingley Road, Cambridge, CB3 OHA, UK

${ }^{13}$ E. A. Milne Centre for Astrophysics, University of Hull, Cottingham Road, Kingston-upon-Hull, HU6 7RX, UK

Accepted XXX. Received YYY; in original form ZZZ

\begin{abstract}
We present early science results from the First Large Absorption Survey in H I (FLASH), a spectroscopically blind survey for 21-cm absorption lines in cold hydrogen (H I gas at cosmological distances using the Australian Square Kilometre Array Pathfinder (ASKAP). We have searched for $\mathrm{HI}$ absorption towards 1253 radio sources in the GAMA 23 field, covering redshifts between $z=0.34$ and 0.79 over a sky area of approximately $50 \mathrm{deg}^{2}$. In a purely blind search we did not obtain any detections of $21-\mathrm{cm}$ absorbers above our reliability threshold. Assuming a fiducial value for the $\mathrm{H}$ I spin temperature of $T_{\text {spin }}=100 \mathrm{~K}$ and source covering fraction $c_{\mathrm{f}}=1$, the total comoving absorption path length sensitive to all Damped Lyman $\alpha$ Absorbers (DLAs; $\left.N_{\mathrm{HI}} \geq 2 \times 10^{20} \mathrm{~cm}^{-2}\right)$ is $\Delta X=6.6 \pm 0.3(\Delta z=3.7 \pm 0.2)$ and superDLAs $\left(N_{\mathrm{HI}} \geq 2 \times 10^{21} \mathrm{~cm}^{-2}\right)$ is $\Delta X=111 \pm 6(\Delta z=63 \pm 3)$. We estimate upper limits on the $\mathrm{H}$ I column density frequency distribution function that are consistent with measurements from prior surveys for redshifted optical DLAs, and nearby $21-\mathrm{cm}$ emission and absorption. By cross-matching our sample of radio sources with optical spectroscopic identifications of galaxies in the GAMA 23 field, we were able to detect $21-\mathrm{cm}$ absorption at $z=0.3562$ towards NVSS J224500-343030, with a column density of $N_{\mathrm{HI}}=(1.2 \pm 0.1) \times 10^{20}\left(T_{\text {spin }} / 100 \mathrm{~K}\right) \mathrm{cm}^{-2}$. The absorber is associated with GAMA J22450.05-343031.7, a massive early-type galaxy at an impact parameter of $17 \mathrm{kpc}$ with respect to the radio source and which may contain a massive $\left(M_{\mathrm{HI}} \gtrsim 3 \times 10^{9} \mathrm{M}_{\odot}\right)$ gas disc. Such gas-rich early types are rare, but have been detected in the nearby Universe.
\end{abstract}

Key words: galaxies:evolution - galaxies: high redshift - galaxies: ISM - galaxies: structure - radio:lines galaxies 


\section{INTRODUCTION}

Star formation and supermassive black hole (SMBH) growth are two important processes in galaxies that influence their evolution throughout cosmic history. However, we do not yet understand why the global rates of star formation (e.g. Hopkins \& Beacom 2006; Madau \& Dickinson 2014; Driver et al. 2018) and SMBH growth (e.g. Ueda et al. 2003; Shankar et al. 2009) both peaked at $z \approx 2$, and then declined by an order magnitude to this epoch. It is clear that a ready supply of cold $\left(T_{\mathrm{k}} \ll 10^{4} \mathrm{~K}\right)$ gas is important; in the nearby Universe the surface densities of star formation and neutral gas, particularly the molecular component, are strongly correlated (e.g. Schmidt 1959; Kennicutt 1998; Bigiel et al. 2008), and likewise radiatively efficient active galactic nuclei (AGNs) are predominantly hosted by star-forming galaxies with a central young stellar population and therefore ample cold gas reservoirs (e.g. Kauffmann et al. 2003, 2007; Kauffmann \& Heckman 2009; LaMassa et al. 2013; Ellison et al. 2019). Determining how the neutral interstellar medium in galaxies has evolved over the history of the Universe is therefore a key component in understanding their evolution. Much of our knowledge of the global content of neutral gas in galaxies comes from observing hydrogen gas, the most common element in the Universe. In its neutral atomic ( $\mathrm{H} \mathrm{I})$ phase, hydrogen is readily detectable in nearby galaxies via $21-\mathrm{cm}$ emission at radio wavelengths (see Giovanelli \& Haynes 2016 for a review) or, at cosmological distances, through Lyman $\alpha(n=1-2)$ absorption in the ultraviolet and visible bands (see Wolfe et al. 2005). The total H I mass density shows comparatively less evolution over cosmological time-scales than that of star formation, decreasing by at most two-fold since $z \approx 2$ (e.g. Zwaan et al. 2005; Martin et al. 2010; Braun 2012; Noterdaeme et al. 2012; Zafar et al. 2013; Crighton et al. 2015; Sánchez-Ramírez et al. 2016; Bird et al. 2017; Rhee et al. 2018), suggesting that much of the neutral gas content of galaxies is replenished over these time-scales.

In contrast, observations of the molecular gas at cosmological distances, traced by emission lines from the low- $J$ rotational transitions of carbon monoxide $\left({ }^{12} \mathrm{C}^{16} \mathrm{O}\right)$, suggest a much stronger evolution of the coldest $\left(T_{\mathrm{k}} \sim 10 \mathrm{~K}\right.$ ) gas (see Carilli \& Walter 2013 for a review). Samples of star-forming and ultraluminous galaxies (e.g. Tacconi et al. 2013; Combes et al. 2013; Magdis et al. 2014; Villanueva et al. 2017; Isbell et al. 2018) have revealed a decrease in the molecular gas fraction in these galaxies since the peak of star formation at $z \approx 2$. Recent deep observations for $\mathrm{CO}$ emission lines in the Hubble Ultra Deep Field with the Atacama Large Millimetre Array (ALMA) also show a decrease in the knee of the CO luminosity function over the same period, implying a corresponding decrease in the cosmological density of $\mathrm{H}_{2}$ that appears to closely match that of the star formation rate (Decarli et al. 2016, 2019).

Radio surveys for the H I $21-\mathrm{cm}$ absorption line, seen in the spectra of radio sources, afford us an important additional tool in establishing a census of the neutral gas in galaxies at cosmological distances (Kanekar \& Briggs 2004; Morganti et al. 2015). 21-cm absorption lines are detected in individual galaxies at luminosity distances well beyond that currently obtainable for emission line surveys ( $z \lesssim 0.4$; Fernández et al. 2016), and are only limited by the observable band of the telescope and a sufficiently large population of detectable background sources. At redshifts below $z=1.7$, the ultraviolet Lyman $\alpha$ line is no longer redshifted into the optical window and can only be observed using the Hubble Space Telescope. Searches for DLAs at these redshifts are therefore observationally expensive and necessarily lead to smaller sample sizes and correspondingly poorer statistical constraints on the cosmological evolution of H I than at higher redshifts (e.g. Neeleman et al. 2016; Rao et al. 2017). To improve the DLA detection rate, it is common practice to select quasars that have existing Mg II $\lambda \lambda 2796,2803 \AA$ absorption with equivalent widths greater than about $0.5 \AA$ (e.g. Rao et al. 2006; Ellison \& Lopez 2009; Rao et al. 2017). There is a concern that this might bias the identification of $\mathrm{H} \mathrm{I}$ absorbers, particularly against those with low column densities and metallicities (e.g. Péroux et al. 2004; Dessauges-Zavadsky et al. 2009; Neeleman et al. 2016; Berg et al. 2017, but see also Rao et al. 2017). The 21-cm absorption line is therefore key to establishing the H I content of galaxies at these intermediate cosmological distances.

Importantly, the equivalent width of the $21-\mathrm{cm}$ absorption line depends on both the H I column density and its excitation (spin) temperature along the line of sight to the radio source. If the $\mathrm{HI}$ column density can be determined independently via either $21-\mathrm{cm}$ emission (e.g. Reeves et al. 2016; Borthakur 2016; Gupta et al. 2018) or Lyman $\alpha$ absorption (see Kanekar et al. 2014 and references therein), then the spin temperature in the absorber can be inferred. However, in doing so one must be careful to consider the relative sizes of the foreground absorber and background continuum source, particularly at radio wavelengths where the source may be significantly larger than the spatial distribution of opaque $\mathrm{H}$ I structures (see e.g. Curran et al. 2005; Braun 2012).

The spin temperature enables comparisons to be drawn with the multiphase neutral interstellar medium (ISM) seen in the Milky Way and Local Group galaxies (e.g. Dickey et al. 1994, 2000; Heiles \& Troland 2003; Roy et al. 2013; Murray et al. 2018), in particular the inferred fraction of cold $\left(T_{\mathrm{k}} \sim 100 \mathrm{~K}\right)$ neutral medium (CNM), which is the component of the atomic gas most likely to trace star formation. Searches for 21-cm absorption in known DLAs suggest that the typical spin temperature of high column density systems is anticorrelated with their metallicity and may increase at redshifts above $z=2$, beyond the peak of star formation in the Universe (Kanekar et al. 2014). This would be consistent with a model whereby relatively metal-poor DLAs in the early Universe (e.g. Rafelski et al. 2012; Cooke et al. 2015; De Cia et al. 2018) lacked sufficient coolants in the gas to form a significant fraction of CNM via fine structure line cooling. Recently, by explicitly modelling the source covering fraction as a function of angular diameter distance, Curran $(2019,2017)$ used literature searches for redshifted $21-\mathrm{cm}$ absorption to show that the spin temperature has evolved with the star formation rate history of the Universe. However, we caution that this uses an evolutionary model for the covering fraction of radio sources that would mimic any perceived evolution in the spin temperature and so future model-independent methods are required to verify such a claim.

Future large-scale, radio-selected $21-\mathrm{cm}$ absorption line surveys will be able to statistically determine the cosmological evolution of the physical state of the neutral atomic gas at intermediate redshifts (e.g. Darling et al. 2011; Allison et al. 2016b). Several of these surveys will be undertaken using pathfinder telescopes to the planned Square Kilometre Array. These include the First Large Absorption Survey in H I (FLASH; e.g. Allison et al. 2016b) with the Australian Square Kilometre Pathfinder (ASKAP; Johnston et al. 2007), the South African MeerKAT Absorption Line Survey (MALS; Gupta et al. 2016) and the Search for H I Absorption with AperTIF (e.g. Oosterloo et al. 2009).

In this paper, we present early science results from ASKAP FLASH, a spectroscopically blind survey for $\mathrm{H}$ I absorption which will eventually cover the entire southern sky $\left(\delta<+10^{\circ}\right)$ between $z=0.4$ and 1.0. Such a survey with ASKAP is enabled by combining a $30 \mathrm{deg}^{2}$ field of view, $300 \mathrm{MHz}$ of instantaneous bandwidth 
Table 1. Summary of observations of the GAMA 23 field using the lower frequency bands of ASKAP-12. Each observation was assigned a unique scheduling block identification (SBID) number and has two interleaved pointing centres labelled A and B. $t_{\text {obs }}$ denotes the duration of the observation. $\sigma_{\text {chan }}$ is the rms noise per $18.5 \mathrm{kHz}$ channel, where we give the interquartile range over the whole bandwidth and all $36 \mathrm{PAF}$ beams. $\Delta v_{\text {chan }}$ is the spectral resolution in rest-frame radial velocity and $z_{\mathrm{HI}}$ is the $\mathrm{H}$ I $21-\mathrm{cm}$ redshift range across the observed band.

\begin{tabular}{|c|c|c|c|c|c|c|c|c|c|c|}
\hline \multirow[t]{2}{*}{ SBID } & \multirow{2}{*}{$\begin{array}{l}\text { Date } \\
\text { UTC }\end{array}$} & \multicolumn{3}{|c|}{ Pointing centre } & \multirow{2}{*}{$\begin{array}{l}\text { Frequency band }^{a} \\
{[\mathrm{MHz}]}\end{array}$} & \multirow[t]{2}{*}{ No. ant. ${ }^{a}$} & \multirow{2}{*}{$\begin{array}{c}t_{\mathrm{obs}} \\
{[\mathrm{h}]}\end{array}$} & \multirow{2}{*}{$\begin{array}{c}\sigma_{\text {chan }} \\
{\left[\mathrm{mJy}_{\text {beam }}^{-1} \text { ] }\right.}\end{array}$} & \multirow{2}{*}{$\begin{array}{c}\Delta v_{\text {chan }} \\
{\left[\mathrm{km} \mathrm{s}^{-1}\right]}\end{array}$} & \multirow{2}{*}{$z_{\mathrm{HI}}$} \\
\hline & & & RA [J2000] & Dec. [J2000] & & & & & & \\
\hline \multirow[t]{2}{*}{2955} & $2016 / 12 / 17$ & A & $23^{\mathrm{h}} 00^{\mathrm{m}} 00.000$ & $-32^{\circ} 30^{\prime} 00^{\prime \prime} 00$ & $864.5-1056.5$ & 12 & 4 & $12-14$ & $5.2-6.4$ & $0.34-0.64$ \\
\hline & & B & $23^{\mathrm{h}} 03^{\mathrm{m}} 01^{\mathrm{s}} .096$ & $-32^{\circ} 29^{\prime} 51^{\prime \prime} 89$ & & & & & & \\
\hline \multirow[t]{2}{*}{2961} & 2016/12/18 & A & $23^{\mathrm{h}} 00^{\mathrm{m}} 00^{\mathrm{s}} .000$ & $-32^{\circ} 30^{\prime} 00^{\prime \prime} 00$ & $864.5-1056.5$ & 12 & 4 & $12-14$ & $5.2-6.4$ & $0.34-0.64$ \\
\hline & & B & $23^{\mathrm{h}} 03^{\mathrm{m}} 01^{\mathrm{s}} .096$ & $-32^{\circ} 29^{\prime} 51^{\prime \prime} 89$ & & & & & & \\
\hline \multirow[t]{2}{*}{4996} & 2018/01/13 & A & $22^{\mathrm{h}} 46^{\mathrm{m}} 13^{\mathrm{s}} .600$ & $-32^{\circ} 15^{\prime} 35^{\prime \prime} .27$ & $792.5-1032.5$ & 15 & 11 & $6.5-8.0$ & $5.4-7.0$ & $0.38-0.79$ \\
\hline & & B & $22^{\mathrm{h}} 48^{\mathrm{m}} 21^{\mathrm{s}} .955$ & $-32^{\circ} 42^{\prime} 31^{\prime \prime} 25$ & & & & & & \\
\hline \multirow[t]{2}{*}{5000} & 2018/01/15 & A & $23^{\mathrm{h}} 11^{\mathrm{m}} 46.399$ & $-32^{\circ} 15^{\prime} 35^{\prime \prime} 27$ & $792.5-1032.5$ & 15 & 11 & $6.8-8.4$ & $5.4-7.0$ & $0.38-0.79$ \\
\hline & & B & $23^{\mathrm{h}} 13^{\mathrm{m}} 54.754$ & $-32^{\circ} 42^{\prime} 31^{\prime \prime} .25$ & & & & & & \\
\hline \multirow[t]{2}{*}{5229} & 2018/03/16 & A & $22^{\mathrm{h}} 46^{\mathrm{m}} 13^{\mathrm{s}} \cdot 600$ & $-32^{\circ} 15^{\prime} 35^{\prime \prime} .27$ & $816.5-1056.5$ & 16 & 11 & $5.4-6.2$ & $5.2-6.8$ & $0.34-0.74$ \\
\hline & & B & $22^{\mathrm{h}} 48^{\mathrm{m}} 21^{\mathrm{s}} .955$ & $-32^{\circ} 42^{\prime} 31^{\prime \prime} 25$ & & & & & & \\
\hline \multirow[t]{2}{*}{5232} & 2018/03/18 & A & $23^{\mathrm{h}} 11^{\mathrm{m}} 46.399$ & $-32^{\circ} 15^{\prime} 35^{\prime \prime} 27$ & $816.5-1056.5$ & 16 & 11 & $6.6-7.7$ & $5.2-6.8$ & $0.34-0.74$ \\
\hline & & B & $23^{\mathrm{h}} 13^{\mathrm{m}} 54.754$ & $-32^{\circ} 42^{\prime} 31^{\prime \prime} .25$ & & & & & & \\
\hline
\end{tabular}

${ }^{a}$ Note that the available frequency band and antennas changed throughout commissioning of the array and correlator.

and a frequency band that below $1 \mathrm{GHz}$ is typically free of any radio frequency interference. Several recent results from observations of radio galaxies and quasars during ASKAP commissioning have demonstrated feasibility (e.g. Allison et al. 2015, 2016a; Moss et al. 2017; Allison et al. 2017; Glowacki et al. 2019; Allison et al. 2019). Here, we have used observations of the $23 \mathrm{hr}$ field of the Galaxy And Mass Assembly survey (GAMA; Liske et al. 2015) ${ }^{1}$, a $50 \mathrm{deg}^{2}$ area of the southern sky that contains spectroscopic information for galaxies to an $i$-band magnitude limit of $i<19.2$ mag. Due to commissioning constraints on the available correlator hardware, our observations covered $\mathrm{H}$ I redshifts between $z_{\mathrm{HI}}=0.34$ and 0.79 , which is slightly different to that expected for FLASH. Our broad goal was to carry out the first wide-field spectroscopically blind search for $21-\mathrm{cm}$ absorption at cosmological distances (see also Darling et al. 2004, 2011) and establish the methodology that will be employed in future, larger surveys.

We structure this paper as follows. In section 2, we describe briefly our observations and data analysis, referring the interested reader to previous work and providing the salient updates. We discuss the results of our survey in section 3 , both when spectroscopically blind and when we cross-match with optically identified galaxies. We summarize our conclusions in section 4. In all distance calculations dependent on the cosmological parameters we adopt a flat lambda cold dark matter $(\Lambda \mathrm{CDM})$ cosmology with $H_{0}=70 \mathrm{~km} \mathrm{~s}^{-1}, \Omega_{\mathrm{m}}=0.3$ and $\Omega_{\Lambda}=0.7$ (e.g. Spergel et al. 2007).

\section{OBSERVATIONS AND DATA}

The GAMA 23 field (Liske et al. 2015) was observed with ASKAP as part of the early science programs of the Evolutionary Map of the Universe survey (EMU; Norris et al. 2011; Leahy et al. 2019) and the Deep Investigation of Neutral Gas Origins survey (DINGO) ${ }^{2}$. These observations used ASKAP-12, a sub-array of ASKAP that had between 12 and 16 operational antennas and a bandwidth between 192 and $240 \mathrm{MHz}$, primarily to carry out early science and commission the Mrk II Phased Array Feeds (PAFs; see e.g. Chippendale et al. 2015). In order to closely match the frequency band

1 http: //www. gama-survey.org

2 https://dingo-survey.org we expect to use with the full FLASH survey (700 - $1000 \mathrm{MHz})$, and to maximize the comoving path for intervening absorption detection, we only chose those observations that used the lowest available band at that time. Three distinct observations were undertaken from 2016 December to 2018 March; we summarize these in Table 1 . The spectral resolution is set by the $18.5 \mathrm{kHz}$ channelization, which for the observed bands corresponds to rest-frame velocities in the range 5.2 to $7.0 \mathrm{~km} \mathrm{~s}^{-1}$. Maximum signal-to-noise PAF beams were electronically formed in a $6 \times 6$ square pattern on the sky with a separation of $0.9 \mathrm{deg}$ between adjacent beam centres. Each observation had two interleaved pointing positions that were switched every $15 \mathrm{~min}$ so as to enable a uniform sensitivity pattern across the field and avoid correlated noise.

A dedicated ASKAPSOFT data processing pipeline is currently being developed for the full FLASH survey, adapting the procedure discussed by Kleiner et al. (2019). However, to facilitate processing of the early science data presented here we have followed the procedure described by Allison et al. (2015, 2017, 2019) and refer the interested reader to that work for further details. The ingested data from the correlator were written to measurement set format and then flagged for digital glitches and autocorrelations, averaged, and split, using the $\mathrm{CASA}^{3}$ package (McMullin et al. 2007). For each PAF beam, we split the data at full spectral resolution into $1-\mathrm{MHz}$ band chunks, equal to the beamforming intervals. Separately, a single $1 \mathrm{MHz}$-averaged data set was produced to be used for high signal-to-noise continuum imaging and self-calibration. Further automated flagging, calibration and imaging of the data was carried out using the MIRIAD ${ }^{4}$ package (Sault et al. 1995). Short observations of PKS B1934-638 (approximately 5 min per PAF beam) were used to obtain initial solutions for the complex antenna gains and to calibrate the flux scale (using the model of Reynolds 1994). We then used the catalogue of the NRAO Very Large Array Sky Survey (NVSS; Condon et al. 1998) to construct a reference sky model for initial self-calibration, followed by iterative self-calibration using deconvolved models from the ASKAP data. Continuum images were formed using multifrequency synthesis with a Brigg's robustness weighting parame-

\footnotetext{
3 http://www.casa.nrao.edu

4 http://www.atnf.csiro.au/computing/software/ miriad/
} 


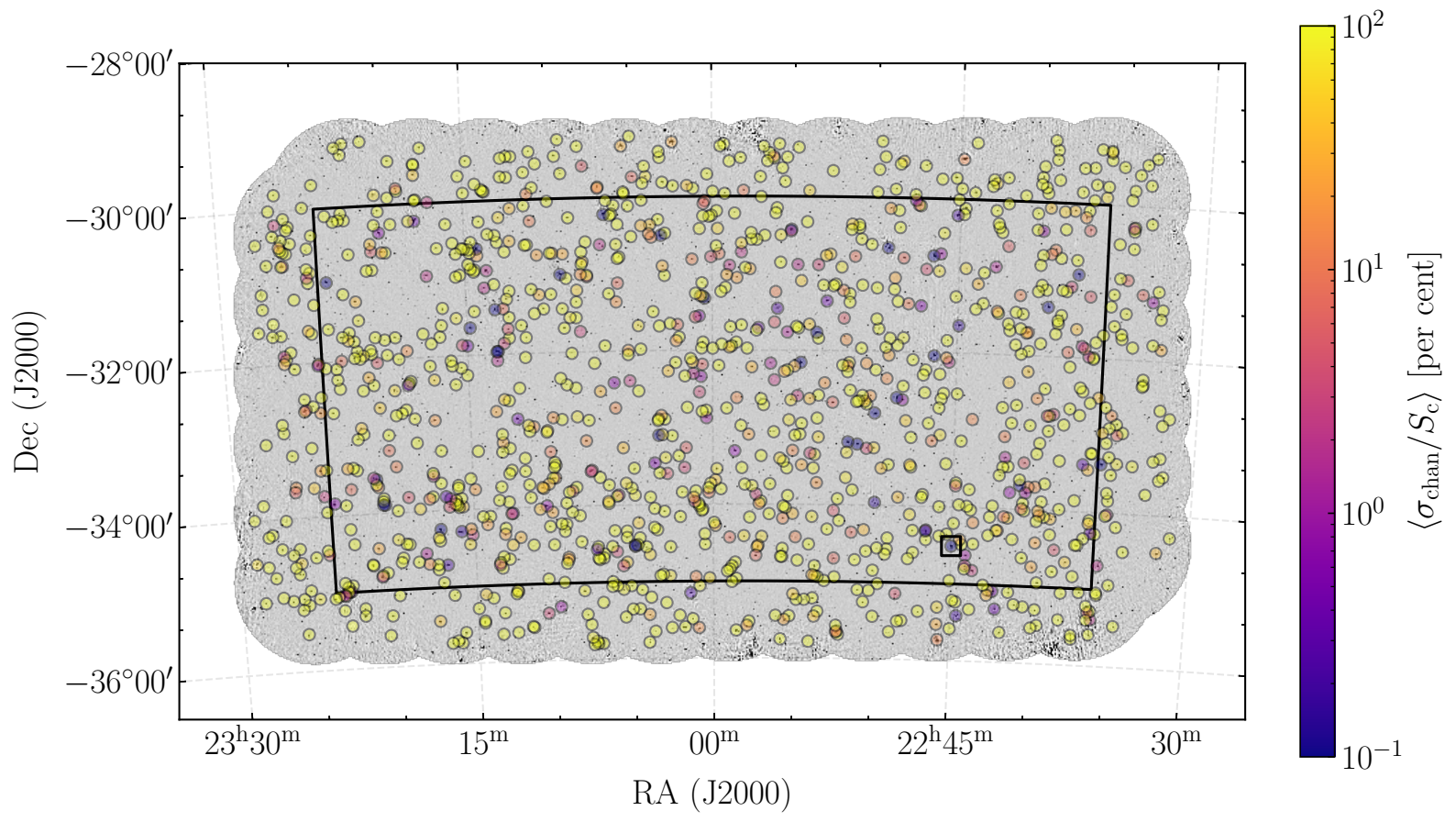

Figure 1. An ASKAP-12 mosaic of the $900 \mathrm{MHz}$ radio continuum in the GAMA 23 field (grey scale image). The small circles indicate the 1253 radio sources towards which we searched for H I 21-cm absorption. The colour scale denotes the median rms noise per $18.5 \mathrm{kHz}$ channel as a fraction of the continuum; darker circles indicate higher sensitivity to $21-\mathrm{cm}$ absorption. The small black square indicates the position of NVSS J224500-343030, towards which we have detected H I 21-cm absorption (see text for details). The larger black boundary denotes the extent of the GAMA 23 field (Liske et al. 2015).

ter of 0.5 , giving an average synthesized beam of $40 \times 25$ arcsec (full width at half maximum; FWHM) with a standard deviation of 10 per cent between the PAF beams and observations. At the median H I $21-\mathrm{cm}$ redshift of our observations $(z=0.57)$, this resolution corresponds to a physical scale of approximately $200 \mathrm{kpc}$. This is not sufficient to accurately determine the ratio of compact to extended radio emission for a given source, and so estimate the areal covering fraction of the $\mathrm{HI}$ distributed in front of the source. The effect of the unknown source covering fraction is to underestimate from the data the true $21-\mathrm{cm}$ optical depth of foreground neutral gas (e.g. Braun 2012). In the subsequent analysis we therefore leave the source covering fraction as a free parameter.

Previous searches for HI absorption with ASKAP were towards individual bright radio sources at the phase centre (e.g. Allison et al. 2015). Here we have carried out a search for H I absorption towards multiple sources across the GAMA 23 field, and so we must also consider the effects of wide-field imaging. For the $1 \mathrm{MHz}$ channel-averaged data, the effect of bandwidth smearing at $900 \mathrm{MHz}$ is comparable to the synthesized beam at angular separations greater than about $5 \mathrm{deg}$ from the phase centre. Likewise, the ASKAP correlator uses $10 \mathrm{~s}$ time-averaging, which leads to more than 10 per cent reduction in source amplitude at separations greater than about $5 \mathrm{deg}$ from the phase centre. Compared with the theoretical half power width of the PAF beam, $\lambda / D_{\text {dish }} \approx 1.6 \mathrm{deg}$, the effects of bandwidth and time-average smearing are therefore negligible. Of greater importance is the effect of non-coplanar antenna baselines; since the INVERT imaging task in MIRIAD does not implement facet gridding (Cornwell \& Perley 1992) or $w$-term projection (Cornwell et al. 2008) to correct for wide-field aberration, we must account for the degree of distortion of sources away from the phase centre in each PAF beam. For a co-planar array, the apparent position shift in arcseconds, caused by the phase er- ror introduced by ignoring the $w$-term, is approximately equal to $2.4 \times 10^{-6} \theta^{2} \sin (z)$, where $\theta$ is the separation from the phase centre in arcseconds and $z$ is the angle from zenith. This error is comparable to the synthesized beam at a separation of about 1 deg from the phase centre and is evident in the continuum images of sources beyond the half-power point of individual PAF beams. This reduces the point source amplitude, and hence sensitivity to $\mathrm{H} \mathrm{I}$ absorption, by approximately 10 per cent across the observed field, but importantly does not introduce any systematic error in our results.

Continuum subtraction was performed on the calibrated, full spectral resolution visibility data in two stages; first we generated a CLEAN component model for each 1-MHz chunk and subtracted it using the MIRIAD task UVMODEL. We then removed residual continuum using the task UVLIN with a second-order polynomial. Since the PAF element weights used to form the beams are applied uniformly in frequency intervals before channelization at full 18.5$\mathrm{kHz}$ resolution, any discontinuous jumps in amplitude and phase are subtracted out through this procedure. However, this necessarily leads to the subtraction of spectral features that have a width greater than the beam forming intervals. Since the early science data presented here were formed in 1-MHz intervals, our results are therefore incomplete to lines broader than approximately $300 \mathrm{~km} \mathrm{~s}^{-1}$. This is large compared with the distribution of line widths for intervening $21-\mathrm{cm}$ absorbers in the literature (see e.g. Curran et al. $2016 \mathrm{~b}$ ), and so unlikely to affect our detecting rate for these systems. However, this could be a factor in detecting intrinsic $21-\mathrm{cm}$ absorbers associated with AGNs, which typically have broader line profiles (see subsection 3.4). For the full FLASH survey, we expect to form the PAF beams on larger $10-\mathrm{MHz}$ intervals, equal to line widths more than a few thousand $\mathrm{km} \mathrm{s}^{-1}$.

The image cubes were generated with natural weighting to optimize sensitivity to absorption line detection, with a subsequent 


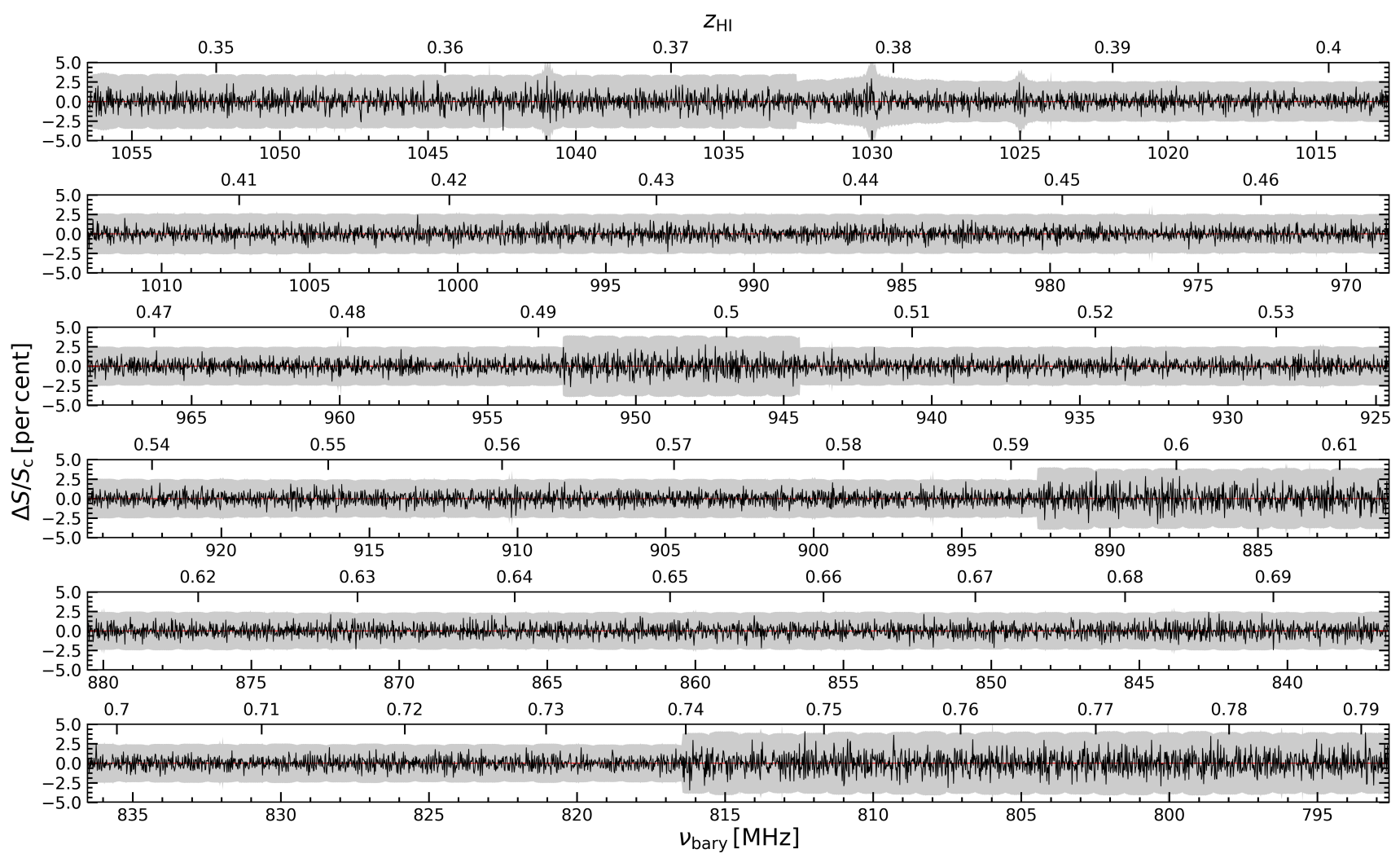

Figure 2. An example ASKAP-12 spectrum towards the radio source NVSS J224500-343030 in the GAMA 23 field. The continuum-subtracted data (black line) are given as a fraction of the continuum flux density. The grey region denotes 5 times the rms noise measured from the channel images. Variations in sensitivity across the band are dominated by observations of different duration at distinct frequencies (see Table 1), and occasional failures of individual correlator cards. On the lower horizontal axis is the solar barycentric-corrected observed frequency and the upper horizontal axis is the corresponding $\mathrm{H}$ redshift.

60 percent reduction in spatial resolution compared with the fullbandwidth continuum images. We summarize the spectral line flux density sensitivity achieved per $18.5-\mathrm{kHz}$ channel for each observation in Table 1, which is consistent with the duration of observation and number of antennas. Some variation in sensitivity between observations is expected given the process of electronically forming the individual PAF beams. Given that the expected final rms noise in the averaged data is $3 \mathrm{mJy}_{\text {beam }}{ }^{-1}$ per $18.5 \mathrm{kHz}$, and assuming a 100 per cent source covering fraction, sources with a flux density greater than $10 \mathrm{mJy}$ are sufficient to detect any absorption at a signal-to-noise ratio $(\mathrm{S} / \mathrm{N})$ of better than 3 . We used the NVSS catalogue (Condon et al. 1998) as a prior to identify the positions of target continuum sources within the field, which is complete for source flux densities greater than $2.5 \mathrm{mJy}$ and has positional accuracy better than 7 arcsec. Although we could have identified target sources using the ASKAP continuum image, the NVSS is matched in spatial resolution ( $45 \mathrm{arcsec})$ to the data cubes and is therefore suitable to use here. For each PAF beam and observation, the spectra were extracted from the data cube at the position of peak source flux density in the corresponding full-bandwidth ASKAP continuum image. Each spectrum was converted to fractional absorption by dividing by the continuum flux density measured as a function of frequency at that position, using continuum images generated from each $1-\mathrm{MHz}$ band-chunk. We then created a single spectrum for the source by averaging over the spectra using an inverse variance weighting to optimize the $\mathrm{S} / \mathrm{N}$.

In Figure 1, we show the full ASKAP-12 image of the 900$\mathrm{MHz}$ continuum in the GAMA 23 field, which we constructed by carrying out a linear mosaic over all observations and PAF beams. In Figure 2, we show an example ASKAP-12 spectrum at the position of NVSS J224500-343030, which demonstrates the spectral fidelity and sensitivity as a function of redshift for these data. The median rms noise in our final spectra is $3.2 \mathrm{mJy}$ beam $^{-1}$ per 18.5 $\mathrm{kHz}$ channel, with a standard deviation of 20 per cent across the observed band and field. We note that this is similar to the expected rms noise in a single $2 \mathrm{~h}$ pointing with the FLASH survey (e.g. Allison et al. 2016b) and so these data are a useful early demonstration of the survey. However, the sensitivity to $\mathrm{HI}$ absorption is a function of the source flux density; of our spectra, 1253 have a median rms noise per $18.5 \mathrm{kHz}$ channel less than 100 per cent fractional absorption of the background continuum, 469 have an rms noise less than 10 per cent and 31 have an rms noise less than 1 per cent - this is summarised for each sightline in Figure 1. In the following analysis, we have used all 1253 spectra with a median rms noise less than 100 per cent fractional absorption, so as to detect all possible absorption lines present in the data. The H I redshift spanned by each spectrum is between $z_{\mathrm{HI}}=0.34$ and 0.79 , but the total absorption search path sensitive to a given minimum column density depends on the redshifts of individual sources, the subtended source flux density, and the $\mathrm{HI}$ spin temperature. We discuss this further in the following analysis. 


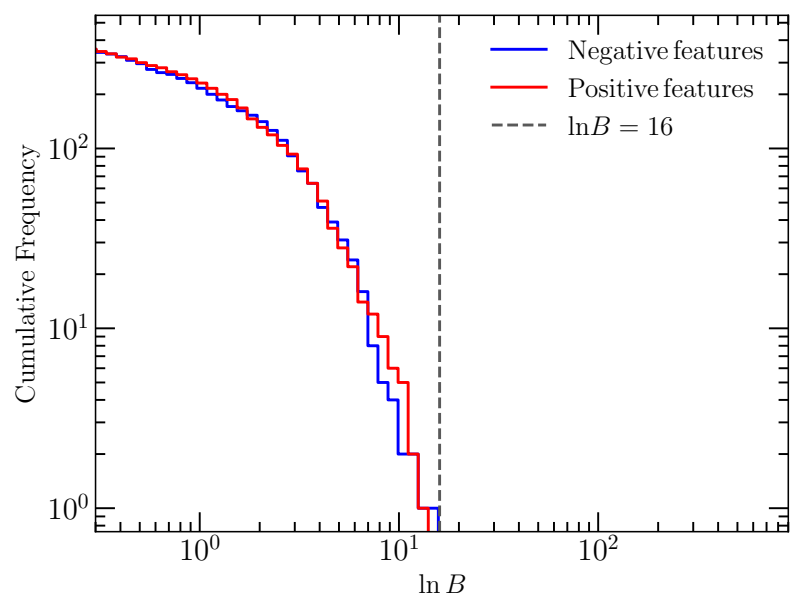

Figure 3. The cumulative frequency distribution of spectral features detected in ASKAP-12 observations of the GAMA 23 field, as a function of the Bayes factor $B$. The blue and red solid lines denote negative and positive spectral features, respectively. Since we do not expect to detect positive features (i.e. emission lines) in our data, we assign a threshold of $\ln B>16$ for reliable detection of absorption (vertical dashed line). As can be seen, above this reliability threshold we do not detect any $\mathrm{H}$ I absorption.

\section{RESULTS AND DISCUSSION}

\subsection{Detection and reliability}

We search for H I absorption in our spectra using an automated detection method based on Bayesian model comparison (see Allison et al. 2012, 2014). This method implements multimodal nested sampling (Feroz \& Hobson 2008; Feroz et al. 2009) to enable multiple redshifted lines to be found in a given spectrum. The significance of each detected feature is given by the Bayes factor $B$ (e.g. Kass \& Raftery 1995), a statistic that is equal to ratio of Bayesian evidences of a Gaussian absorption-line model and a null model, which contains no line. This assumes no prior preference for either model, which is reasonable if we are testing for the incidence of absorption lines. We note that this method of feature detection is model dependent, but that it is reasonable to expect that extragalactic 21-cm absorption will comprise one or more Gaussian velocity distributions. We use the likelihood function for a normal distribution, with standard deviation equal to the measured rms noise in each channel. The following non-informative priors are used for each model parameter: for the line position we use a uniform prior over the full range of the spectrum, for the FWHM we use a loguniform prior between 0.1 and $2000 \mathrm{~km} \mathrm{~s}^{-1}$, and for the peak optical depth we use a loguniform prior between 1 percent of the median rms noise and a maximum value of 10 . These ranges are chosen to include reasonable boundaries set by the data and physically realistic limits.

Since the data are sparsely populated by an unknown distribution of absorption lines, we expect that the noise will necessarily produce a distribution of spurious detections. We therefore need to establish a reliability threshold for $B$ above which we are confident a given detected feature is real. Given the sensitivity of our data ${ }^{5}$ we do not expect to detect any real H I 21-cm emission lines and

5 The rms noise per channel in our spectra is (3.2 \pm 0.6$) \mathrm{mJy} \mathrm{beam}^{-1}$, which for an unresolved galaxy at the lowest redshift of $z=0.34$ gives a $5-\sigma$ detection limit on 21-cm emission that corresponds to an $\mathrm{H} \mathrm{I}$ mass of $M_{\mathrm{HI}} \sim$ $4.1 \times 10^{11} \mathrm{M}_{\odot}$ (e.g. Meyer et al. 2017). This is beyond the extreme high-

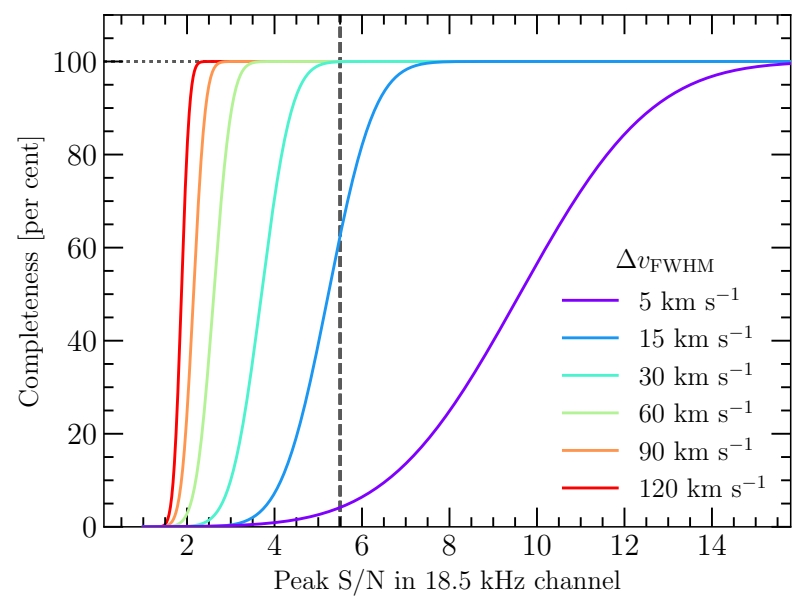

Figure 4. The expected completeness of reliable detections in our data as a function of the peak $\mathrm{S} / \mathrm{N}$ in a single $18.5-\mathrm{kHz}$ channel and the FWHM of the optical depth velocity distribution. The vertical dashed line denotes the peak signal-to-noise ratio at which we recover all lines with FWHM velocity width equal to $\Delta \nu_{\text {FWHM }}=30 \mathrm{~km} \mathrm{~s}^{-1}$; the mean width for intervening 21$\mathrm{cm}$ absorbers detected in the literature (see e.g. Curran et al. 2016b; Allison et al. 2016b).

can therefore use the distribution of positive features to determine this reliability threshold (see e.g. Serra et al. 2012a). In Figure 3 we plot the cumulative frequency distribution of both negative and positive features as a function of the Bayes factor. A threshold of $\ln (B)>16$ is found to be sufficient for a reliable detection of absorption in our data ${ }^{6}$. Based on this reliability criterion we do not detect any $\mathrm{H}$ I absorption in a blind search of our data.

\subsection{Sensitivity to HI absorption}

We calculate the $21-\mathrm{cm}$ optical depth sensitivity of each spectral element (i.e. $18.5-\mathrm{kHz}$ channel) in our data using

$\int \tau_{21} \mathrm{~d} v>0.57\left[\frac{\tau_{\text {peak }}}{0.018}\right]\left[\frac{\Delta v_{\text {FWHM }}}{30 \mathrm{~km} \mathrm{~s}^{-1}}\right] \mathrm{km} \mathrm{s}^{-1}$,

where $\Delta v_{\text {FWHM }}$ is the FWHM rest-frame velocity, assuming a Gaussian distribution, and $\tau_{\text {peak }}$ is the peak optical depth sensitivity. For a fixed velocity width, $\tau_{\text {peak }}$ is given by

$\tau_{\text {peak }}=-\ln \left(1-0.018\left[\frac{\mathrm{S} / \mathrm{N}}{5.5}\right]\left[\frac{\sigma_{\text {chan }}}{3.2 \mathrm{mJy} \mathrm{bm}^{-1}}\right]\left[\frac{S_{\mathrm{c}} c_{\mathrm{f}}}{1 \mathrm{Jy} \mathrm{bm}^{-1}}\right]^{-1}\right)$,

where $\mathrm{S} / \mathrm{N}$ is the minimum peak signal-to-noise ratio required for recovery of absorption lines to high completeness, $\sigma_{\text {chan }}$ is the rms noise, $S_{\mathrm{c}}$ is the background continuum flux density and $c_{\mathrm{f}}$ is the unknown areal fraction of the unresolved source covered by foreground H I. In the following, we assume that most 21-cm absorbers

mass end of the local H I mass function (Jones et al. 2018) and therefore we do not expect any detections of 21-cm emission.

6 The reliability of our ASKAP-12 data is limited by a multiplicative nonGaussian contribution to the noise at the level of 1 per cent. This was due to incorrect firmware weights used in correcting for the coarse channelisation of the data. This error has now been corrected and does not affect data obtained with the full 36-antenna ASKAP. 
are in the optically thin regime $\left(\tau_{\text {peak }}<<1\right)$, so that the peak optical depth is inversely proportional to the source covering fraction and we explicitly give our results as a function of a single unknown; the spin temperature to source covering fraction ratio, $T_{\text {spin }} / c_{\mathrm{f}}$. However, it should be noted that for the rarest optically thick $21-\mathrm{cm}$ absorbers this approximation does not hold and the source covering fraction should be included in the natural logarithm in Equation 2.

We estimate the required $\mathrm{S} / \mathrm{N}$ in Equation 2 by randomly populating our spectra with 1000 fake absorption lines and calculating the fraction recovered using our detection method and reliability threshold. In Figure 4, we show the results for velocity widths ranging from $\Delta v_{\mathrm{FWHM}}=5$ to $120 \mathrm{~km} \mathrm{~s}^{-1}$. As expected, narrower lines are less complete for a fixed peak sensitivity, but more complete for a fixed total optical depth sensitivity. Since the sensitivity does depend on the choice of velocity width, we adopt a value equal to the mean for all intervening 21-cm absorbers detected in the literature, $\Delta v_{\text {FWHM }}=30 \mathrm{~km} \mathrm{~s}^{-1}$ (see e.g. Curran et al. 2016b; Allison et al. 2016b). In this case, we recover all lines that have a peak S/N greater than 5.5, and that is the value we adopt in the following analysis.

The H I column density sensitivity of each spectral element is then given by

$N_{\mathrm{HI}}>1.0 \times 10^{20}\left[\frac{T_{\text {spin }}}{100 \mathrm{~K}}\right]\left[\frac{\tau_{\text {peak }}}{0.018}\right]\left[\frac{\Delta v_{\mathrm{FWHM}}}{30 \mathrm{~km} \mathrm{~s}^{-1}}\right] \mathrm{cm}^{-2}$,

where the spin temperature $\left(T_{\text {spin }}\right)$ is the column-density-weighted harmonic mean for all phases of the HI gas along the line of sight through the absorber. In the case of the Galactic ISM, the harmonic mean spin temperature is $T_{\text {spin }} \approx 300 \mathrm{~K}$ with three $\mathrm{H} \mathrm{I}$ phases consisting of cold (CNM; $T_{\text {spin }} \approx 100 \mathrm{~K}$ ), unstable (UNM; $T_{\text {spin }} \approx 500 \mathrm{~K}$ ) and warm neutral medium (WNM; $T_{\text {spin }} \approx 10^{4} \mathrm{~K}$ ), in mass fractions of 28,20, and 52 per cent, respectively (Murray et al. 2018). In general, the mass fraction of phases is expected to vary depending on the physical conditions within each absorber (see e.g. Kanekar et al. 2014 and references therein). For comparison with previous work in the literature we adopt a fiducial value of $T_{\text {spin }}=100 \mathrm{~K}$, but also show results for $T_{\text {spin }}=1000 \mathrm{~K}$.

We next estimate the total comoving absorption path length $(\Delta X)$ spanned by our data, by summing over all spectral channels that are sensitive to a minimum column density given by Equation 3 . For the standard $\Lambda \mathrm{CDM}$ cosmology, the comoving absorption path element for the $i$ 'th channel is given by

$\Delta X_{i}=\Delta z_{i}\left(1+z_{i}\right)^{2} E\left(z_{i}\right)^{-1}$,

where

$E\left(z_{i}\right)=\sqrt{\left(1+z_{i}^{3}\right) \Omega_{\mathrm{m}}-\left(1+z_{i}\right)^{2}\left(\Omega_{\mathrm{m}}+\Omega_{\Lambda}-1\right)+\Omega_{\Lambda}}$,

$\Delta z_{i}=\left(1+z_{i}\right) \frac{\Delta v_{\text {chan }}}{v_{i}}$

$z_{i}=\frac{v_{\mathrm{HI}}}{v_{i}}-1$,

$v_{i}$ is the solar barycentric-corrected centre frequency of the $i$ 'th channel, $\Delta v_{\text {chan }}$ is the channel separation, equal to $18.5 \mathrm{kHz}$, and $v_{\mathrm{HI}}$ is the rest frequency of the $\mathrm{HI} 21-\mathrm{cm}$ line, equal to 1420.40575177 MHz (Hellwig et al. 1970).

The comoving absorption path length covered by each source spectrum depends on the cosmological redshift of that source, which in some cases may be within the range spanned by our data

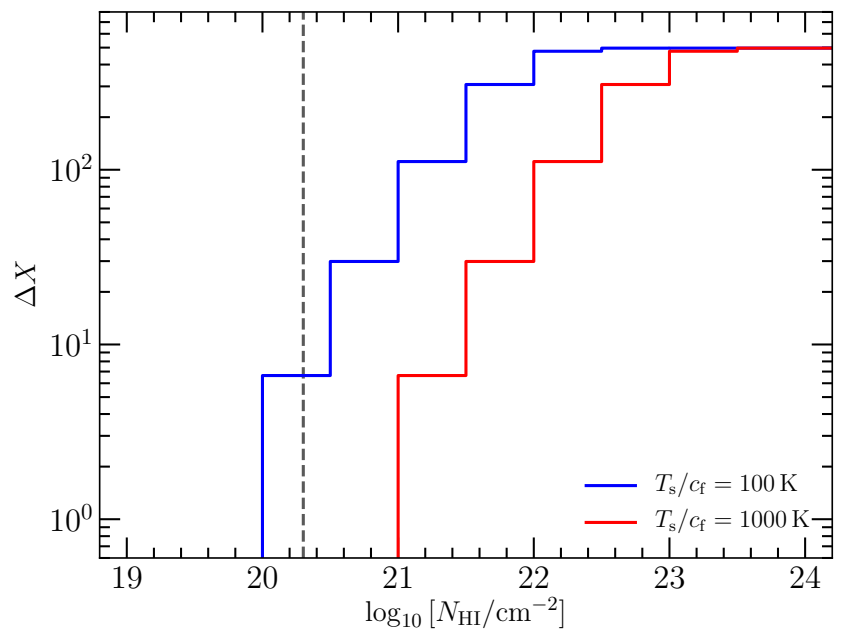

Figure 5. The comoving absorption path length $(\Delta X)$ spanned by our data as a function of $\mathrm{H}$ I column density sensitivity. We give results for spin temperature to source covering fraction ratios of 100 (blue line) and $1000 \mathrm{~K}$ (red line). The vertical dashed line indicates the lower limit definition for DLAs $\left(N_{\mathrm{HI}} \geq 2 \times 10^{20} \mathrm{~cm}^{-2}\right)$.

or even at a lower redshift. We account for this by weighting each $\Delta X_{i}$ by the probability that the source lies at a cosmological redshift greater than the spectral channel. We exclude absorption associated with H I gas in the host galaxy of the radio source by shifting the channel redshift used in the weighting by a rest-frame radial velocity of $\Delta v_{\text {asc }}=3000 \mathrm{~km} \mathrm{~s}^{-1}$. The total comoving absorption path length is thus given by the following sum over all spectral channels that are sensitive to a given minimum column density

$\Delta X=\sum_{i} w_{i} \Delta X_{i}$

where

$w_{i}=w\left(z_{i}+\Delta z_{\mathrm{asc}, i}\right)$,

$\Delta z_{\mathrm{asc}, i}=\left(1+z_{i}\right) \Delta v_{\mathrm{asc}} / c$,

and $w$ is the redshift weighting function.

We do not a priori use optical matching to confirm redshifts for individual sources, which would be both highly incomplete for our large sample and prone to confusion error. Instead we use a statistical weighting for all sources based on the redshift distribution given by De Zotti et al. (2010) from a fit to bright $\left(S_{1.4} \geq 10 \mathrm{mJy}\right.$ ) sources in the Combined EIS-NVSS Survey Of Radio Sources (CENSORS; Brookes et al. 2008). In this case, the weighting function used in Equation 9 is given by

$w(z)=\frac{\int_{z}^{\infty} \mathcal{N}_{\text {src }}\left(z^{\prime}\right) \mathrm{d} z^{\prime}}{\int_{0}^{\infty} \mathcal{N}_{\text {src }}\left(z^{\prime}\right) \mathrm{d} z^{\prime}}$,

where the redshift distribution is given by (De Zotti et al. 2010)

$\mathcal{N}_{\text {src }}(z)=1.29+32.37 z-32.89 z^{2}+11.13 z^{3}-1.25 z^{4}$.

For the $\mathrm{HI}$ redshifts covered by our data, the fractional uncertainty in the cumulative redshift distribution due to the size of the CENSORS sample is approximately $3-6$ percent. This translates directly to the same fractional uncertainty in our estimates of the total comoving absorption path length and expected detection rates for the survey. 


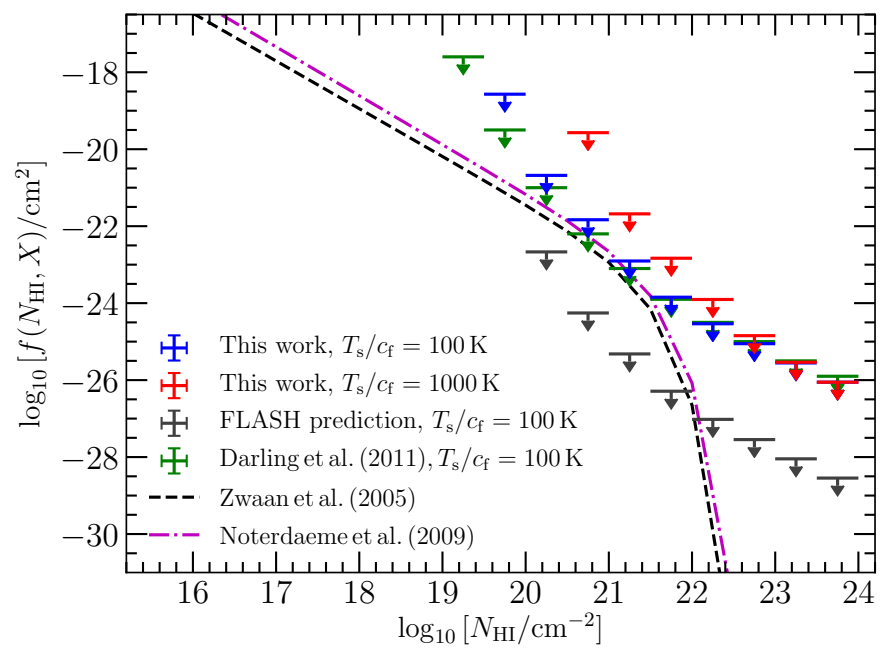

Figure 6. 95 per cent upper limits on the H I column density frequency distribution from our ASKAP-12 observations of the GAMA23 field. We give distributions for values of the spin temperature to source covering fraction ratio of $100 \mathrm{~K}$ (blue points) and $1000 \mathrm{~K}$ (red points). We predict the sensitivity of the planned FLASH survey (assuming $T_{\text {spin }} / c_{\mathrm{f}}=100 \mathrm{~K}$ ) by scaling the integration time $(2 \mathrm{~h})$, number of antennas (36), sky area (south of $\delta=+10 \mathrm{deg}$ ) and redshift coverage $(z=0.4$ to 1.0$)$. Also shown are results from the ALFALFA absorption pilot survey by Darling et al. (2011) and model fits to data given by Zwaan et al. (2005) at $z=0$ and Noterdaeme et al. (2009) at $z \approx 3$.

In Figure 5 we show the total comoving absorption path length as a function of $\mathrm{HI}$ column density. We show results for $T_{\text {spin }} / c_{\mathrm{f}}=100$ and $1000 \mathrm{~K}$, which are indicative values spanning that typically measured for sight lines through the Milky Way interstellar medium (e.g. Heiles \& Troland 2003; Murray et al. 2018). The total comoving absorption path length spanned by our data is $\Delta X=500 \pm 25$, and for $T_{\text {spin }} / c_{\mathrm{f}}=100 \mathrm{~K}$ the total path sensitive to all DLAs is $\Delta X=6.6 \pm 0.3$ (redshift interval of $\Delta z=3.7 \pm 0.2$ ) and super-DLAs $\left(N_{\mathrm{HI}} \geq 2 \times 10^{21} \mathrm{~cm}^{-2}\right)$ is $\Delta X=111 \pm 6(\Delta z=63 \pm 3)$.

\subsection{Limits on the $N_{\mathrm{HI}}$ frequency distribution function and spin temperature}

As can be seen in Figure 5, our data vary by several orders of magnitude in $\mathrm{H}$ I column density sensitivity and as a function of the spin temperature and source covering fraction. To compare our results with previous 21-cm and DLA surveys for $\mathrm{HI}$ gas, it is therefore necessary that we determine the sensitivity of our data to the frequency of intervening systems as a function of $\mathrm{H}$ I column density and $T_{\mathrm{spin}} / c_{\mathrm{f}}$. Upper limits on the $N_{\mathrm{HI}}$ frequency distribution function, $f\left(N_{\mathrm{HI}}, X\right)$, are given by

$f\left(N_{\mathrm{HI}}, X\right)<\frac{\lambda_{\max }}{\Delta N_{\mathrm{HI}} \Delta X}$,

where $\lambda_{\max }$ is the Poisson upper limit on the detection rate of absorbers with column density $N_{\mathrm{HI}}$ in interval $\Delta N_{\mathrm{HI}}$ and $\Delta \mathrm{X}$ is the total comoving absorption path length sensitive to $N_{\mathrm{HI}}$. We use $\Delta N_{\mathrm{HI}}=0.5$ dex and the 95 per cent upper limit on the Poisson rate given by $\lambda_{\max }=3$ when no detections are obtained. This allows us to compare our limits directly with that of Darling et al. (2011), who carried out a blind 21-cm absorption survey of the nearby Universe using pilot data from the Arecibo Legacy Fast Arecibo $L$-band Feed Array (ALFALFA) survey (Giovanelli et al. 2005).

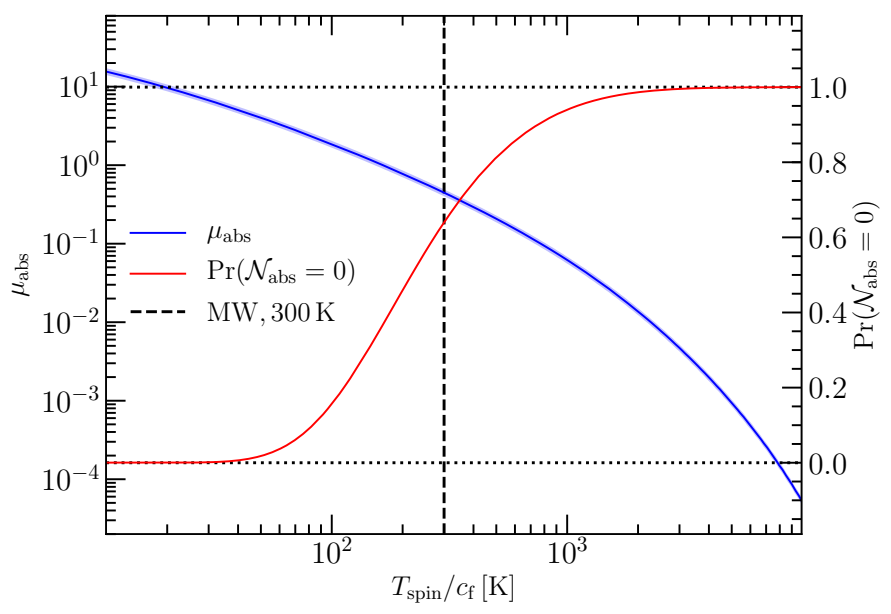

Figure 7. The expected number of reliable detections of $21 \mathrm{~cm}$ absorbers in our data $\left(\mu_{\mathrm{abs}}\right)$, and the corresponding Poisson probability of detecting no absorbers, as a function of the spin temperature to source covering fraction ratio. For comparison, also shown is the harmonic mean spin temperature of the Milky Way ISM, assuming $c_{\mathrm{f}}=1$ (Murray et al. 2018).

We show our upper limits on $f\left(N_{\mathrm{HI}}, X\right)$ in Figure 6, for spin temperature to source covering fraction ratios of $T_{\text {spin }} / c_{\mathrm{f}}=100$ and $1000 \mathrm{~K}$. For super-DLA column densities $\left(N_{\mathrm{HI}} \geq 2 \times 10^{21} \mathrm{~cm}^{-2}\right)$ these are similar to that of Darling et al. (2011), but less sensitive at lower column densities due to the relative flux density sensitivity of the two surveys. We also show the expected sensitivity for the all-sky FLASH survey, scaling to the expected integration time per pointing ( $2 \mathrm{~h}$ ), the number of antennas (36), the sky area (south of $\delta=+10 \mathrm{deg})$ and the redshift coverage $\left(z_{\mathrm{HI}}=0.4\right.$ to 1.0$)$. At DLA column densities, FLASH is expected to be two orders of magnitude more sensitive to $f\left(N_{\mathrm{HI}}, X\right)$ than these early results.

For $T_{\text {spin }} / c_{\mathrm{f}} \gtrsim 100 \mathrm{~K}$, our upper limits on $f\left(N_{\mathrm{HI}}, X\right)$ are consistent with the measured $\mathrm{H}$ I column density frequency distributions from surveys of 21-cm emission in nearby galaxies (Zwaan et al. 2005) and distant $(z \approx 3)$ DLAs (Noterdaeme et al. 2009). Using these measured $f\left(N_{\mathrm{HI}}, X\right)$, we can calculate the expected number of $21-\mathrm{cm}$ absorber detections in our data, given by

$\mu_{\mathrm{abs}}=\sum_{i}\left[\int_{N_{\min }}^{\infty} f\left(N_{\mathrm{HI}}, X_{i}\right) \mathrm{d} N_{\mathrm{HI}}\right] w_{i} \Delta X_{i}$,

where $N_{\min }$ is the minimum column density sensitivity given by Equation 3. We interpolate $f\left(N_{\mathrm{HI}}, X\right)$ over the redshift range covered by our observations; since the total $\mathrm{H}$ I content of the Universe is not expected to evolve strongly at these redshifts, our results are not sensitive to the degree of interpolation employed. The typical measurement uncertainty in $f\left(N_{\mathrm{HI}}, X\right)$, due to the finite sample sizes of galaixes, is 10 per cent over the range of column densities to which our ASKAP data are sensitive. Combining this with the uncertainty in $w(z)$ due to the source redshift distribution, we estimate a fractional uncertainty of 11 per cent in $\mu_{\mathrm{abs}}$.

In Figure 7, we show the expected number of $21-\mathrm{cm}$ absorber detections as a function of $T_{\text {spin }} / c_{\mathrm{f}}$, and the corresponding Poisson probability of obtaining zero detections. For the harmonic mean spin temperature of the Milky Way ISM, $T_{\text {spin }} \approx 300 \mathrm{~K}$ (assuming $c_{\mathrm{f}}=1$ ), the probability of detecting zero absorbers is 64 percent. Our data are consistent with a mean spin temperature of $\mathrm{H}$ I gas at intermediate cosmological distances $(z \sim 0.5)$ equal to the Milky Way ISM, but with a significant non-zero probability for colder gas; 
the likelihood of $T_{\text {spin }} / c_{\mathrm{f}} \lesssim 100 \mathrm{~K}$ for our data is 16 per cent. Any evolution in the CNM fraction at these redshifts will be confirmed by future all-sky surveys that should provide an order of magnitude stronger constraint on the spin temperature as a function of redshift (see e.g. Allison et al. 2016b).

\subsection{Intrinsic 21-cm absorbers}

In addition to intervening galaxies, 21-cm absorption is also detectable from $\mathrm{H}$ I gas in the host galaxy of the radio source (see Morganti \& Oosterloo 2018 for a review). Since we did not detect any $21-\mathrm{cm}$ absorbers within the volume of our survey, it is instructive to compare this result with the incidence of detections reported in recent large-scale targeted surveys for intrinsic $21-\mathrm{cm}$ absorbers. The expected detection rate of intrinsic absorbers in active galaxies is not well defined because of the complex relationship between the physical conditions of the neutral gas in the host galaxy and the AGN. In particular, there is a factor of $\sim 6$ discrepancy between the detection rates in nearby objects and those at higher redshifts (e.g. Curran et al. 2008; Curran \& Whiting 2010, 2012; Aditya et al. 2016; Aditya \& Kanekar 2018; Curran et al. 2019; Grasha et al. 2019). The lower detection rates of intrinsic absorbers in highredshift objects are thought to be due to selection bias - from either UV-luminous quasars that ionize the gas or excitation of the gas by $21-\mathrm{cm}$ photons from the radio source - and possibly also intrinsic redshift evolution in the host galaxy population. In the future, planned wide-field radio-selected surveys using the SKA and its precursors will be key in studying the H I content of these distant radio-AGN hosts (Morganti et al. 2015).

Here we compare our results with that of the radio-selected survey by Maccagni et al. (2017). Using the Westerbork Synthesis Radio Telescope (WSRT), they carried out a search for intrinsic H I absorption in 248 nearby $(z<0.25)$ radio galaxies with core flux densities $S_{1.4}>30 \mathrm{mJy}$, reporting a detection rate of $(27 \pm$ $5.5)$ per cent for their whole sample, with an average peak optical depth sensitivity of approximately $\tau_{\text {peak }} \approx 0.05$. The FWHM of line profiles in intrinsic $21-\mathrm{cm}$ absorbers are typically broader, with an average value of $\Delta v_{\text {FHWM }} \approx 120 \mathrm{~km} \mathrm{~s}^{-1}$ (see e.g. Geréb et al. 2015; Curran et al. 2016b; Maccagni et al. 2017). For these broader line profiles our detection threshold reliably recovers all absorption lines with a peak $\mathrm{S} / \mathrm{N}$ greater than 3 in a single $18.5 \mathrm{kHz}$ channel (see Figure 4). Of the 1253 radio sources in our sample, 54 sight lines have a median rms optical depth noise per spectral channel less than the 1.67 per cent required to detect $\tau_{\text {peak }}=0.05$ at $\mathrm{S} / \mathrm{N}=$ 3.

Given that the sources in our sample are at higher redshifts than that of Maccagni et al. (2017), for the same optical depth sensitivity we select for higher radio luminosity. It is possible that this might select against detection of absorption if the $\mathrm{H}$ I gas is excited to higher spin temperatures by $21-\mathrm{cm}$ photons from the radio source (Bahcall \& Ekers 1969). Of the 54 sightlines in our data that have optical depth sensitivity comparable to that of Maccagni et al. the mean and maximum 1.4-GHz flux densities are $\left\langle S_{1.4}\right\rangle=340 \mathrm{mJy}$ and $\max \left(S_{1.4}\right)=1.3 \mathrm{Jy}$. At the highest redshift covered by our survey, $z=0.79$, these flux densities correspond to radio luminosities equal to $L_{1.4}=5.5 \times 10^{26}$ and $2.1 \times 10^{27} \mathrm{~W} \mathrm{~Hz}^{-1}$, respectively. The sample of Maccagni et al. (2017) spanned comparatively lower luminosities between $L_{1.4}=3.2 \times 10^{22}$ and $1.6 \times 10^{26} \mathrm{~W} \mathrm{~Hz}^{-1}$, indicating that there could be a bias. However, Aditya \& Kanekar (2018) obtained a similar detection rate of $28_{-8}^{+10}$ per cent to Maccagni et al. for a sample of 46 intermediateredshift $(z<1.2)$ compact flat-spectrum radio sources with max- imum radio luminosity $\max \left(L_{1.4}\right)=5.0 \times 10^{27} \mathrm{~W} \mathrm{~Hz}^{-1}$. Furthermore, in their targeted survey of 145 compact radio sources, spanning luminosities in the range $L_{1.4}=1.6 \times 10^{25}$ and $4.5 \times$ $10^{28} \mathrm{~W} \mathrm{~Hz}^{-1}$, Grasha et al. (2019) found no evidence for a statistical correlation between their detection rate and radio luminosity. Similarly, Curran et al. (2019) carried out a study of intrinsic absorbers reported in the literature and found no conclusive evidence that the radio luminosity has a strong effect on the detection rate. Therefore, our results are unlikely to be biased against detecting $21-\mathrm{cm}$ absorbers as a result of our selection for optical depth sensitivity.

Using the global redshift distribution of sources brighter than $10 \mathrm{mJy}$ given by De Zotti et al. (2010), we expect approximately 25 per cent of our sources to be within the volume bounded by $z=$ 0.34 to 0.79 . This means that of the 54 sufficiently sensitive sight lines, approximately 14 are expected to be suitable to search for intrinsic 21-cm absorption. As discussed in section 2, our continuum subtraction process removes spectral artefacts on scales greater than the beam forming interval, so that our search is highly incomplete to lines wider than approximately $300 \mathrm{~km} \mathrm{~s}^{-1}$. Maccagni et al. (2017) find that approximately 25 per cent of their absorption lines have a full-width at 20 per cent maximum (FW20) greater than this velocity width. Assuming a detection rate equal to 27 per cent, and taking into account an additional missing 25 percent of lines, the binomial probability of detecting exactly zero absorbers in our data is $\operatorname{Pr}\left(\mathcal{N}_{\text {abs }}=0\right) \approx 0.04$.

There is therefore tension between non-detection of intrinsic absorbers in our data and the detection rate for the whole sample of Maccagni et al. (2017). An important factor in determining the detection rate of intrinsic absorbers is the morphology of the radio source; simply because compact radio sources are more likely to be located within their host galaxy and subtended by higher column densities of absorbing gas (e.g. Pihlström et al. 2003; Curran et al. 2013). Indeed, Maccagni et al. (2017) report detection rates of $(32 \pm 7.9)$ percent for the 131 compact sources versus $(16 \pm 6.8)$ per cent for the 108 extended sources in their sample, using a morphological classification based on the major-to-minor axis ratio in NVSS (Condon et al. 1998) and the peak-to-integrated flux density ratio in the Faint Images of the Radio Sky at Twenty Centimeters (FIRST; Becker et al. 1995). The lower detection rate reported for extended radio sources is more consistent with our data, with a probability of non-detection $\operatorname{Pr}\left(\mathcal{N}_{\mathrm{abs}}=0\right) \approx 0.17$. Since Maccagni at al. selected sources based on the radio core flux density, primarily so as to study the kinematics of $\mathrm{H} \mathrm{I}$ absorption, this will bias their whole sample towards higher detection rates. Our results show that a lower rate of detection for intrinsic $21-\mathrm{cm}$ absorbers is expected from future wide-field radio-selected surveys, although by how much is yet to be determined.

\subsection{Cross-matching with optically identified galaxies}

Beyond a simple spectroscopically blind survey of the GAMA 23 field, we cross-matched all 1253 radio sources in our full sample against the existing spectroscopic catalogue of galaxies in this field (Driver et al. in preparation). For each radio source, we determine a matched galaxy if: (a) it has a spectroscopic redshift within the range for $\mathrm{H}$ I covered by the ASKAP-12 spectrum, and (b) within an impact parameter of $50 \mathrm{kpc}$, which is the maximum galactocentric radius typically seen for H I discs (e.g. Wang et al. 2016, see also Bland-Hawthorn et al. 2017) and within which 21-cm absorption is expected to be detected (e.g. Reeves et al. 2016; Curran et al. 2016a; Borthakur 2016; Dutta et al. 2017). Note that we do 


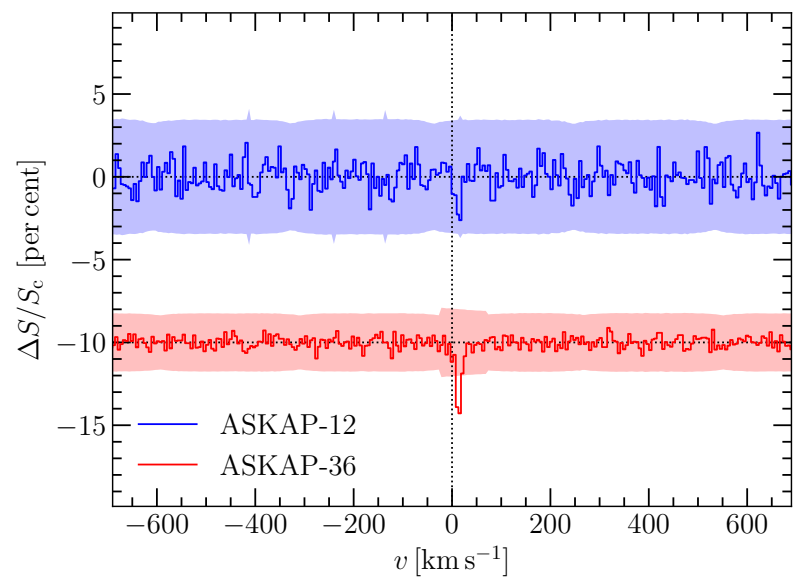

Figure 8. ASKAP spectra towards the radio source NVSS J224500-343030 at the spectroscopically determined redshift $(z=0.3562)$ of the galaxy GAMA J224500.05-343031.7. The detection of $\mathrm{H} \mathrm{I}$ absorption evident in the ASKAP-12 spectrum was later confirmed using the ASKAP-36 array. The continuum-subtracted data (solid lines) are given as a fraction of the continuum flux density. The shaded regions denote 5 times the rms noise measured from the channel images. For clarity the ASKAP 36 spectrum has been offset by 10 per cent.

not use available optical photometry to predetermine the presence and/or size of any H I disc, opting instead for a minimally informative prior based on just the presence of an optical galaxy with a reliable redshift. Based on these criteria, we obtain 51 successful matches between radio sources and GAMA galaxies. The impact parameters range from 0.8 to $50 \mathrm{kpc}$ over a roughly uniform distribution, with 15 matches within $10 \mathrm{kpc}$. We then re-run our line finding method using a normal prior for the line position given by the GAMA redshift.

Of these 51 cross-matches, we obtain one tentative detection of H I absorption towards the radio source NVSS J224500-343030 $\left(S_{843}=587.3 \pm 17.7 \mathrm{mJy}\right.$; Mauch et al. 2003$)$ at the redshift of the galaxy GAMAJ224500.05-343031.7 $(z=0.356$; Driver et al. in preparation). The Bayes factor for the single-component Gaussian model versus noise-only model was $\ln (B)=3.9 \pm 0.1$, with a corresponding two-model probability for the spectral line of $\operatorname{Pr}(M \mid D)=98.0(1)$ per cent. This detection was then confirmed by carrying out a further $6 \mathrm{~h}$ observation on 2019 May 17 (SBID 8808) with the full 36-antenna array (ASKAP-36), achieving an rms noise of $2.1 \mathrm{mJy}^{-1}$ beam ${ }^{-1}$ per $18.5 \mathrm{KHz}$ channel with a continuum spatial resolution at the centre of the band of $22 \times 16$ arcsec. This observation used just a single PAF beam centred on the target source. We show both the ASKAP-12 and ASKAP-36 spectra for NVSS J224500-343030 in Figure 8, centred at the redshift of the galaxy. We obtain $\ln (B)=107.2 \pm 0.1$ for the singlecomponent Gaussian model and find that further complexity with a two-component model is disfavoured with $\Delta \ln (B)=-1.8 \pm 0.1$. As an average across the unresolved continuum flux density, we estimate a peak optical depth of $\tau_{\text {peak }}=0.043 \pm 0.004$, width of $\Delta v_{\text {FHWM }}=(14.1 \pm 1.6) \mathrm{km} \mathrm{s}^{-1}$ and an integrated optical depth of $\int \tau_{21} \mathrm{~d} v=(0.64 \pm 0.06) \mathrm{km} \mathrm{s}^{-1}$. The corresponding H I column density is $N_{\mathrm{HI}}=(1.2 \pm 0.1) \times 10^{20}\left(T_{\text {spin }} / 100 \mathrm{~K}\right) \mathrm{cm}^{-2}$, demonstrating that inclusion of a prior optical identification has enabled us to detect absorption below the sensitivity limit of our blind survey (as shown in Figure 5).

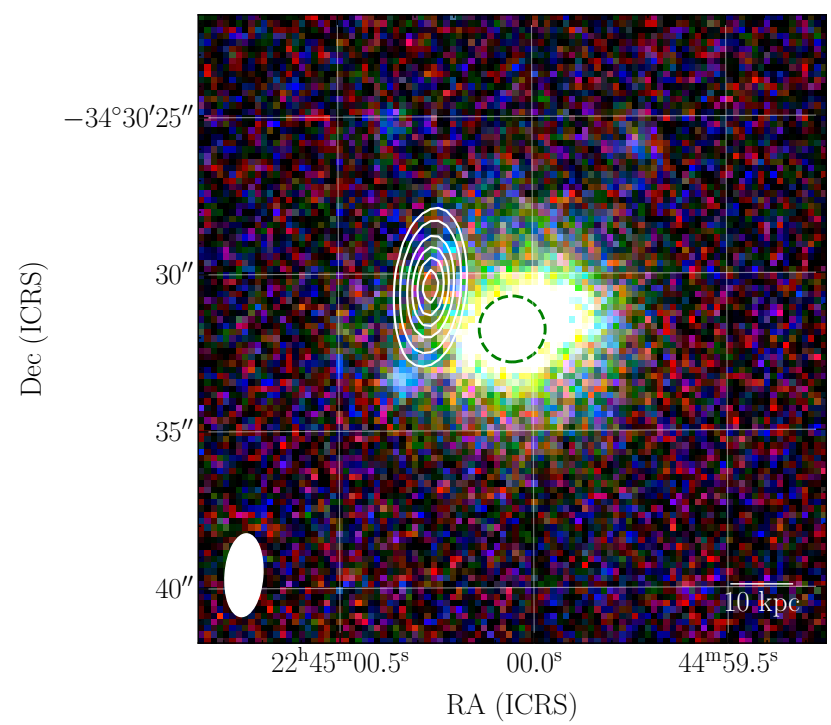

Figure 9. A three-colour optical image of GAMA J224500.05-343031.7, constructed using gri-band images from Data Release 4 of the VST KiloDegree Survey (KiDS; Kuijken et al. 2019). The dashed green circle indicates the position and width of the GAMA spectroscopic aperture. The white contours show the $9.5 \mathrm{GHz}$ continuum from ATCA observations of NVSS J224500-343030 (levels are 5, 10, 20, 30, 40 and $50 \mathrm{mJy}^{\text {beam }}{ }^{-1}$ ). The ATCA restoring beam is shown in the bottom left hand corner. The horizontal bar indicates the physical scale at the redshift of the galaxy.

\subsection{H I absorption in GAMA J224500.05-343031.7}

Using ASKAP we have detected H I absorption at $z=0.3562$ in the galaxy GAMA J224500.05-343031.7. In Figure 9 we show a three-colour optical image of this galaxy using gri-band images from Data Release 4 of the VST Kilo-Degree Survey (KiDS; Kuijken et al. 2019). The catalogued position of the radio source NVSS J224500-343030 is offset from the centre of the galaxy by 2.6 arcsec, which is significant when compared with the uncertainty due to the noise $(\approx 26$ mas $)$ and calibration in NVSS $(\approx 0.5$ arcsec; Condon et al. 1998), and the known astrometric error of the VLA with respect to optical surveys ( $\approx 20$ mas; e.g. Helfand et al. 2015). At the redshift of the galaxy this offset is equivalent to a physical separation of $13 \mathrm{kpc}$ and beyond the half-light radius of $10 \mathrm{kpc}$ (1.9 arcsec), suggesting that the radio source is not associated with the galaxy nucleus. This interpretation is supported by the spectral behaviour of the source, which is characteristic of a compact radio galaxy (see Figure 10). It has a steep power-law spectrum at frequencies above $1.3 \mathrm{GHz}$, with a spectral index of $\alpha \approx-1.2$, that significantly flattens to $\alpha \approx-0.1$ at lower frequencies, likely due to synchrotron self-absorption associated with a characteristic scale of $d_{\text {src }} \lesssim 10 \mathrm{kpc}$ (e.g. Snellen et al. 2000). This suggests that the HI absorption in GAMA J224500.05-343031.7 is seen towards a background radio source (with a radio luminosity $L_{5 \mathrm{GHz}} \gtrsim 2 \times 10^{42} \mathrm{erg} \mathrm{s}^{-1}$ ).

In order to confirm that the source is a background AGN, we use higher resolution data of the radio continuum at 5.5 and $9.5 \mathrm{GHz}$ from the GAMA Legacy ATCA Southern Survey (GLASS; Huynh et al. in preparation) ${ }^{7}$. Observations were carried out using the Australia Telescope Compact Array (ATCA) in

7 https://research.csiro.au/glass/ 


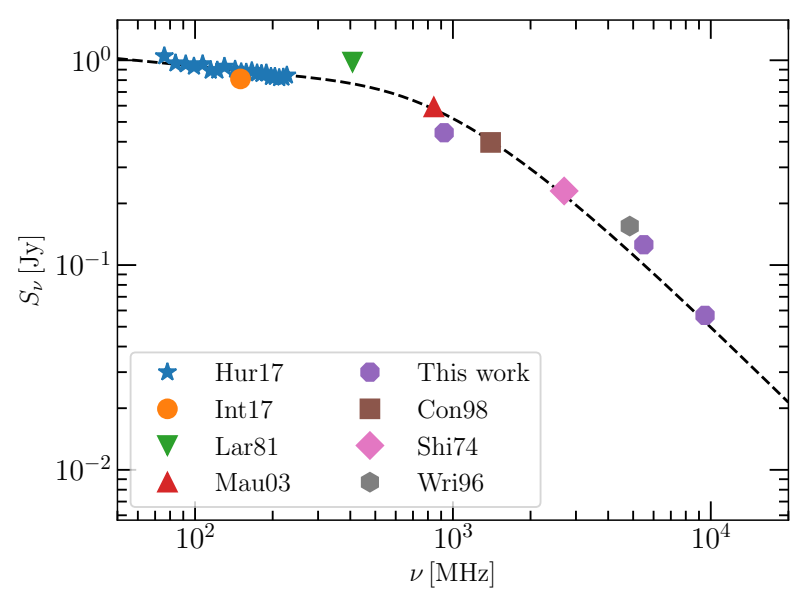

Figure 10. The spectral energy distribution (SED) at radio wavelengths of the source NVSS J224500-343030, compiled using data from the literature data this work. The frequency axis is given in the observer rest frame. The dashed line denotes a best-fitting model that includes optically thick and thin power-law spectra. References for the data: Hurl17 - Hurley-Walker et al. (2017); Int17 - Intema et al. (2017); Lar81 - Large et al. (1981); Mau03 - Mauch et al. (2003); Con98 - Condon et al. (1998); Shi74 - Shimmins \& Bolton (1974); Wri96 - Wright et al. (1996).

$5 \times 40$ s scans on 2019 May 13 under OPAL $^{8}$ project code C3132. The array was in $1.5 \mathrm{~B}$ configuration, which for a Brigg's robustness weighting parameter of 0.5 gave an effective resolution of $4.8 \times 2.0$ and $2.6 \times 1.2$ arcsec at 5.5 and $9.5 \mathrm{GHz}$, respectively. The visibility data were flagged, calibrated, and imaged using standard tasks from MIRIAD. The 5.5 and $9.5-\mathrm{GHz}$ images are consistent with a point source at this resolution, giving an upper limit on the physical size of the source at the galaxy of $\sim 6-13 \mathrm{kpc}$. Using the task IMFIT to fit source models to the images, we obtain flux densities of $S_{5.5}=125.7 \pm 0.4$ and $S_{9.5}=56.8 \pm 0.9 \mathrm{mJy}$. At the lower frequency end of our observations we also estimate a flux density of $S_{4.85}=140.6 \pm 0.4 \mathrm{mJy}$, which is consistent with the total flux density of the source $S_{4.85}=155 \pm 15 \mathrm{mJy}$ measured by the Parkes-MIT-NRAO surveys (PMN; Wright et al. 1996) ${ }^{9}$. The highest resolution $9.5 \mathrm{GHz}$ image is shown in Figure 9 and is consistent with an offset of 3.4 arcsec, which at the redshift of the galaxy equates to a physical separation of about $17 \mathrm{kpc}$. This confirms that the radio source is not associated with the nucleus and is likely a background AGN.

The optical spectrum of GAMAJ224500.05-343031.7, shown in Figure 11, was produced by the GAMA survey using a 2.1-arcsec aperture from the 2dF/AAOmega facility (Saunders et al. 2004; Smith et al. 2004; Sharp et al. 2006) on the 3.9-m Anglo-Australian Telescope (AAT). Observations were carried out on 2012 November 13 and November 17, with a total exposure time of $4100 \mathrm{~s}$ and a median seeing of $0.7 \mathrm{arcsec}$. The original GAMA spectroscopic data for this source were found to have poor spectral reduction due to a strong splicing artefact between the blue and red spectral arm (a known issue in some GAMA spectra, see Hopkins et al. 2013). This artefact does not affect the redshift measurement, which was given a probability match of 99.89 percent, with a corresponding GAMA quality flag of $n Q=4$. None the

\footnotetext{
8 https://opal.atnf.csiro.au

9 https://www.parkes.atnf.csiro.au/observing/

databases/pmn/pmn.html
}

less, for this work the raw spectroscopic data were extracted from the GAMA data base, each arm independently re-flux-calibrated using the $g$ - and $r$-band broad-band photometry and the arms recombined; resulting in the spectrum displayed in Figure 11. This re-reduced spectrum was then independently redshifted using the AUTOZ code (Baldry et al. 2014), and a high confidence redshift confirmed at $z=0.3562$. It is characteristic of an early-type galaxy, exhibiting a $4000 \AA$ A break, $\mathrm{Na}, \mathrm{Mg}$, Ca II H and $\mathrm{K}$ absorption lines, and no detected emission lines.

In Figure 12 we show the spectral energy distribution of the galaxy using photometric data from the GAMA Panchromatic Data Release (Bellstedt et al. in preparation). SED template fitting was carried out using PROSPECT (Robotham et al. 2020), which combines stellar population synthesis models by Bruzual \& Charlot (2003), attenuates the resulting stellar light using the dust model of Charlot \& Fall (2000) and simultaneously fits the expected dust emission in the far infrared using the model of Dale et al. (2014). We parametrize the modelled star formation history as a skewednormal function, described by four free parameters. These free parameters specify at what age the star formation peaks, the peak star formation rate, the "peakiness" of the star formation history, and the skewness of the star formation history. We do not include a separate star formation burst. We fit for the final metallicity of the galaxy, where the metallicity evolution follows the build-up of stellar mass. We estimate a total stellar mass for the galaxy of $M_{\star}=2.85_{-0.34}^{+0.39} \times 10^{11} \mathrm{M}_{\odot}$ and a star formation rate, averaged over the last $100 \mathrm{Myr}$, of SFR $=6.3_{-4.3}^{+21.2} \mathrm{M}_{\odot} \mathrm{yr}^{-1}$.

The wavelength of the far infrared (FIR) peak of $\lambda \sim 3.5 \times$ $10^{6} \AA$ is longer than the typical range of $\lambda \sim(0.7-2.0) \times 10^{6} \AA$ for a galaxy at the given redshift, indicating the possibility that the FIR is being contaminated by the background source at a redshift $z \gtrsim 1.4$. To ensure that the potentially-contaminated FIR flux is not impacting the derived stellar mass and SFR, we repeat the SED-fitting without the FIR bands. We obtain values of $M_{\star}=3.28_{-0.61}^{+5.31} \mathrm{M}_{\odot}$ and SFR $=3.3_{-2.5}^{+55.6} \mathrm{M}_{\odot} \mathrm{yr}^{-1}$, which are consistent within uncertainty with the parameters derived when including the FIR flux, albeit with a significantly larger range.

The $\mathrm{H} \alpha$ line is redshifted beyond the observed spectrum, but using the relationship given by Kewley et al. (2004) we predict from the SFR a luminosity range for the [O II] $\lambda 3727$ emission line of $L_{[\mathrm{OII}]} \sim(0.12-8.9) \times 10^{42} \mathrm{erg} \mathrm{s}^{-1}$, which at the redshift of the galaxy is equivalent to a flux of $S_{[\mathrm{OII}]} \sim(2.8-210) \times$ $10^{-16} \mathrm{erg} \mathrm{s}^{-1} \mathrm{~cm}^{-2}$. This should be detectable in the optical spectrum shown in Figure 11 and non-detection could either be due to dust obscuration or to a large fraction of the star formation occurring outside of the spectroscopic aperture. From the dust attenuation at $\lambda=5000 \AA$ shown in Figure 12, it is possible that the line flux density is attenuated by up to 75 percent. This is potentially sufficient to obscure the expected [O II] $\lambda 3727$ emission, particularly at the lower end of the predicted flux range. However, we also note that evidence for concentrated star formation is seen in the colour image in Figure 9 at the edges of the galaxy (blue regions), and the presence of high column densities of cold $(T \sim 100 \mathrm{~K}) \mathrm{H} \mathrm{I}$ gas at a similar galactocentric radius would be consistent with this scenario.

At a galactocentric radius of $17 \mathrm{kpc}$, the integrated optical depth of the absorption line, $\int \tau_{21} \mathrm{~d} v=(0.64 \pm 0.06) \mathrm{km} \mathrm{s}^{-1}$, is consistent with the spatial distribution of other intervening $21-\mathrm{cm}$ absorbers reported in the literature (see e.g. Curran et al. 2016a; Dutta et al. 2017). If we assume that the spin temperature is greater than or equal to $100 \mathrm{~K}$, then the lower limit H I column density is 


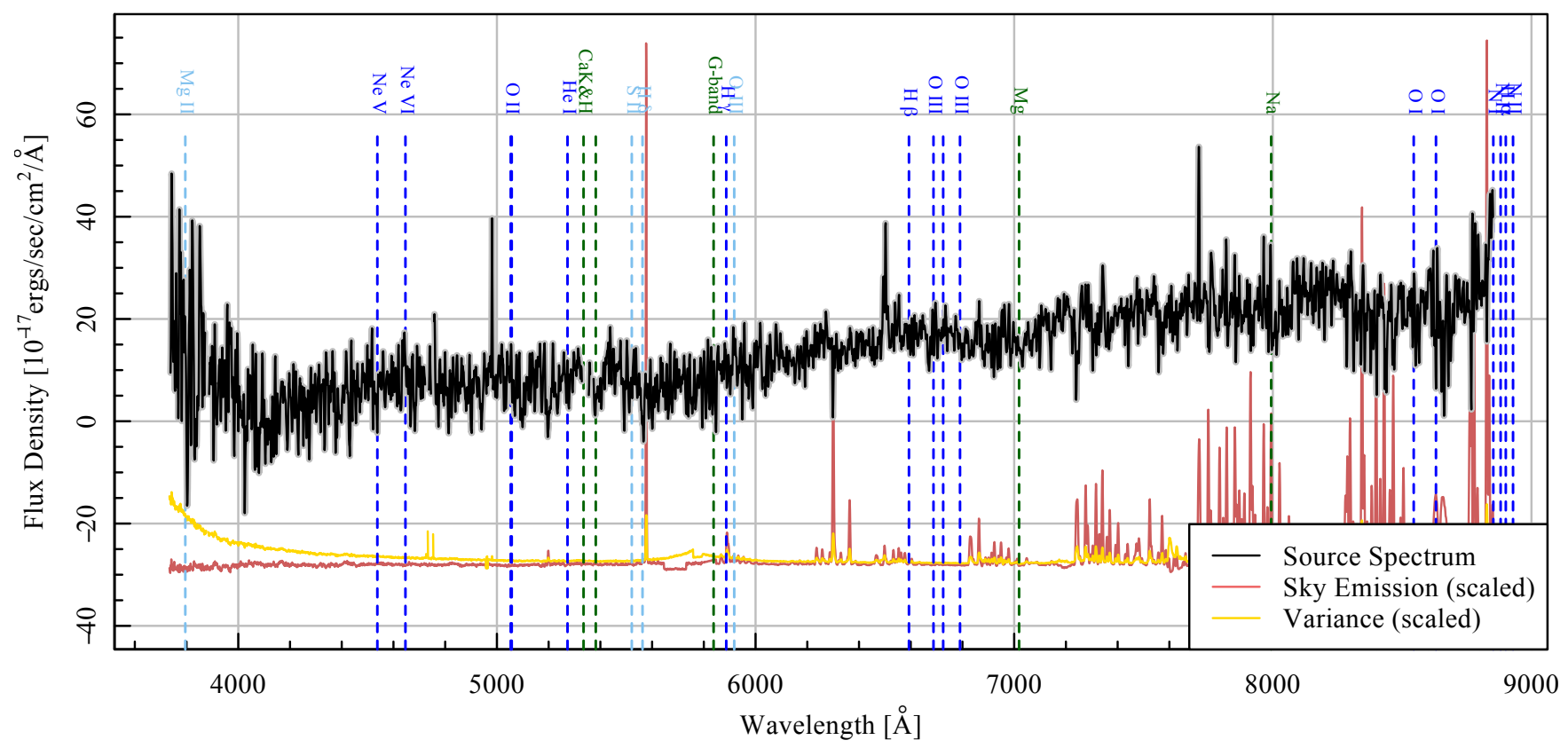

Figure 11. Optical spectrum from the central 2.1 arcsec of the intervening galaxy GAMA J224500.05-343031.7. The spectrum, smoothed for visual clarity using a 9th-order Hanning window, is consistent with an early-type galaxy at a redshift of $z=0.3562$. The expected positions of spectral lines are as indicated by the vertical dashed lines; dark blue denotes common emission lines, green denotes common absorption lines and light blue denotes lines commonly associated with AGNs.

$N_{\mathrm{HI}} \geq(1.2 \pm 0.1) \times 10^{20} \mathrm{~cm}^{-2}$, with a corresponding surface density of $\Sigma_{\mathrm{HI}} \gtrsim(0.96 \pm 0.1) \mathrm{M}_{\odot} \mathrm{pc}^{-2}$. This is the average value across the unresolved background source, which at the distance of the galaxy has a physical size $d_{\text {src }} \lesssim 10 \mathrm{kpc}$. In their study of H I $21-\mathrm{cm}$ absorption around galaxies at $z<0.4$, Dutta et al. (2017) found that most 21-cm absorbers do not trace the dusty stellar disc, but are aligned with the major axis and so are likely coplanar with any extended $\mathrm{H}$ I disc. If the absorber detected in GAMA J224500.05343031.7 does form part of a larger H I disc, then the H I sizemass relation (see e.g. Wang et al. 2016 and references therein) would imply a total HI mass of $M_{\mathrm{HI}} \gtrsim 3 \times 10^{9} \mathrm{M}_{\odot}$. Massive and extended $\mathrm{HI}$ discs are rare in early type galaxies, but have been seen in the nearby Universe (Serra et al. 2012c). We may therefore be seeing 21-cm absorption through such an $\mathrm{HI}$ disc in GAMA J224500.05-343031.7.

\section{CONCLUSIONS}

Using the ASKAP-12 sub-array of the Australian SKA Pathfinder we have carried out the first widefield spectroscopically blind 21$\mathrm{cm}$ absorption survey at cosmological distances in the GAMA 23 field, covering redshifts between $z=0.34$ and 0.79 over a sky area of $50 \mathrm{deg}^{2}$.

In a blind search of the data towards 1253 radio sources we did not detect any $21-\mathrm{cm}$ absorbers. For a fiducial spin temperature of $T_{\text {spin }}=100 \mathrm{~K}$ and a source covering fraction of $c_{\mathrm{f}}=1$, we find that our data are sensitive to intervening $21-\mathrm{cm}$ absorption in DLAs over a comoving absorption path length $\Delta X \approx 6.6 \pm 0.3$, and for super-DLAs $\left(N_{\mathrm{HI}} \geq 2 \times 10^{21} \mathrm{~cm}^{-2}\right) \Delta X \approx 111 \pm 6$. The 95 per cent upper limits on the $N_{\mathrm{HI}}$ frequency distribution function, $f\left(N_{\mathrm{HI}}, X\right)$, are consistent with that measured using 21 -cm emission-line surveys at $z \approx 0$ (e.g. Zwaan et al. 2005) and DLA surveys at $z \approx 3$ (e.g. Noterdaeme et al. 2009, 2012), and with the 21-cm absorption survey at $z \approx 0$ by Darling et al. (2011). We calculate the probability of obtaining zero detections as a function of $T_{\mathrm{spin}} / c_{\mathrm{f}}$ and find that this result is expected with a probability of 64 percent if the harmonic mean spin temperature is equal to that in the Milky Way $\left(T_{\text {spin }} \approx 300 \mathrm{~K}\right.$; Murray et al. 2018).

We also find that this result is in tension with the detection rates of previous targeted surveys for intrinsic $21-\mathrm{cm}$ absorbers in nearby radio galaxies. If we assume a detection rate equal to 27 per cent, based on the complete sample of Maccagni et al. (2017), we find that the probability of detecting no intrinsic absorbers in our data is $\operatorname{Pr}\left(\mathcal{N}_{\mathrm{abs}}=0\right)=0.04$. Given that previous surveys for intrinsic absorbers have selected targets based on their compact flux density, we suggest that the detection rates for future large-scale wide-field surveys are likely to be lower and closer to that found for extended radio galaxies.

By cross-matching the positions of radio sources in our data with optical galaxies that were spectroscopically identified to be within the volume, we were able to search for $21-\mathrm{cm}$ absorption at a lower significance than the Bayes factor threshold used for detection in our blind search. We detected H I absorption at $z=0.3563$ towards the radio source NVSS J224500-343030, later confirming this using more sensitive observations with the full 36-antenna ASKAP. The absorption line is consistent with the spectroscopic redshift $(z=0.3562)$ of GAMA J224500.05-343031.7, an intervening early-type galaxy at an impact parameter of $17 \mathrm{kpc}$. Analysis of the photometric data, available through the GAMA survey of this field, reveals star formation over the past $100 \mathrm{Myr}$ at the level of $\mathrm{SFR} \sim 0.5-4 \mathrm{M}_{\odot} \mathrm{yr}^{-1}$. Visual inspection of the gri-band images shows localized star formation in the outskirts of the galaxy at the same galactocentric radius as the absorber. We suggest that the 21$\mathrm{cm}$ absorber, with an H I column density $N_{\mathrm{HI}} \gtrsim 1.2 \times 10^{20} \mathrm{~cm}^{-2}$, 


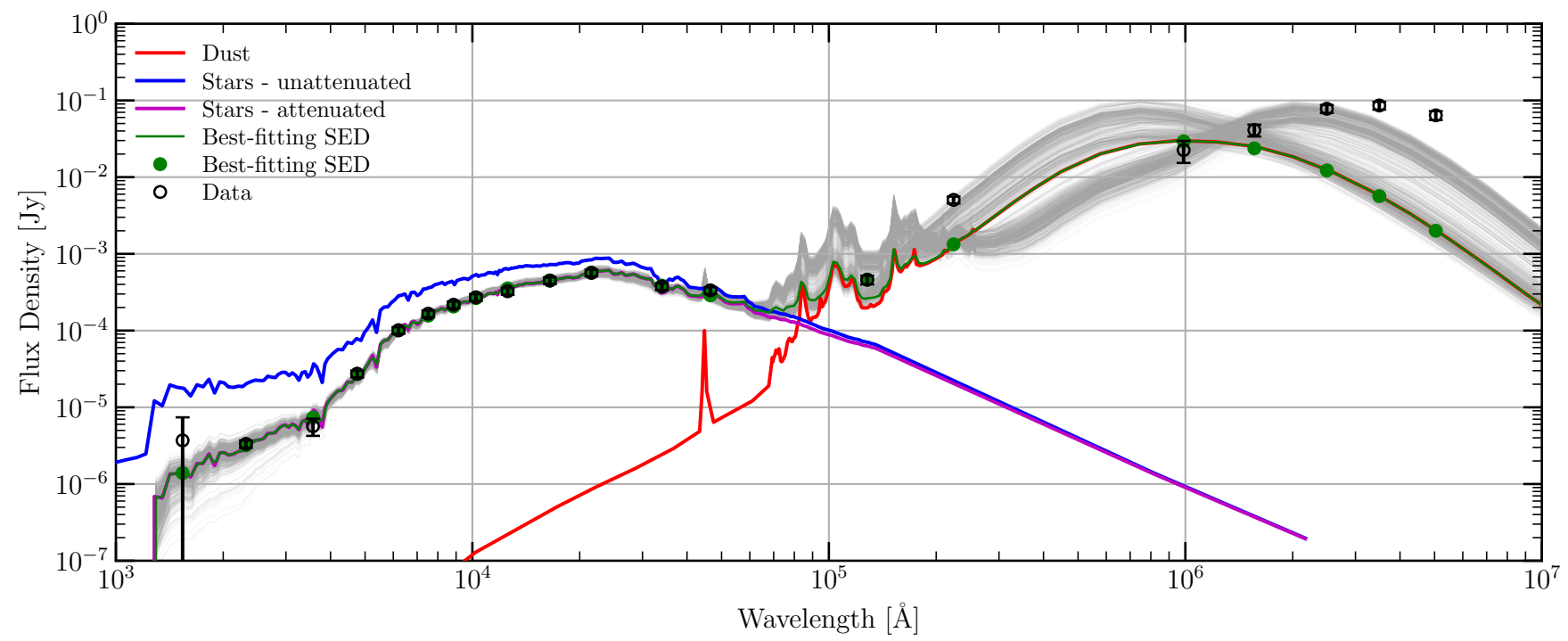

Figure 12. The SED of the intervening galaxy GAMA J224500.05-343031.7. The photometric data shown were compiled using the GAMA Panchromatic Data Release (Bellstedt et al. in preparation). The data (grey points) were extracted from each image using PRoFound (Robotham et al. 2018). Template fitting (coloured lines) was carried out using PROSPECT (Robotham et al. 2020). The blue line denotes the best-fitting stellar light using the population synthesis models of Bruzual \& Charlot (2003). The magenta line is the best-fitting stellar light after attenuating the blue line using the dust model of Charlot $\&$ Fall (2000). The red line is the best-fitting dust emission in the far infrared using the model of Dale et al. (2014). Grey lines show 1000 randomly sampled iterations from the MCMC chain, indicating the sampled range of the model SED.

may form part of a massive $\mathrm{H}$ I disc similar to that seen in some gasrich early type galaxies in the nearby Universe (Serra et al. 2012b).

Our results demonstrate the feasibility of carrying out a widefield wide-band survey for 21-cm absorption with ASKAP. The optical spectroscopic coverage of the GAMA23 field allowed us to detect H I 21-cm absorption below our blind detection threshold. However, we predict that for $\mathrm{H}$ I column densities greater than DLA-like systems $\left(N_{\mathrm{HI}} \geq 2 \times 10^{20} \mathrm{~cm}^{-2}\right)$, the planned ASKAP FLASH survey of the southern sky $\left(\delta<+10^{\circ}\right)$ should be two orders of magnitude more sensitive to the $\mathrm{H}$ I frequency distribution function than these early results, yielding several hundred blind detections of intervening $21-\mathrm{cm}$ absorbers.

\section{ACKNOWLEDGEMENTS}

We dedicate this paper to the memory of Richard (Dick) Hunstead, a wonderful colleague, friend and mentor.

We thank Raffaella Morganti and O. Ivy Wong for their helpful comments on an earlier version of the manuscript. We also thank the anonymous referee and the scientific editor for their comments that helped improve this paper. JRA acknowledges support from a Christ Church Career Development Fellowship. Parts of this research were conducted by the Australian Research Council Centre of Excellence for All-sky Astrophysics in 3D (ASTRO 3D) through project number CE170100013. The National Radio Astronomy Observatory is a facility of the National Science Foundation operated under cooperative agreement by Associated Universities, Inc.

The Australian SKA Pathfinder and the Australia Telescope Compact Array are part of the Australia Telescope National Facility which is managed by CSIRO. Operation of ATCA is funded by the Australian Government. Operation of ASKAP is funded by the Australian Government with support from the National Collaborative Research Infrastructure Strategy. ASKAP uses the resources of the Pawsey Supercomputing Centre. Establishment of ASKAP, the Murchison Radio-astronomy Observatory and the Pawsey Supercomputing Centre are initiatives of the Australian Government, with support from the Government of Western Australia and the Science and Industry Endowment Fund. We acknowledge the Wajarri Yamatji people as the traditional owners of the Observatory site.

The optical images in this work are based on observations made with ESO Telescopes at the La Silla Paranal Observatory under programme IDs 177.A-3016, 177.A-3017, 177.A-3018 and 179.A-2004, and on data products produced by the KiDS consortium. The KiDS production team acknowledges support from: Deutsche Forschungsgemeinschaft, ERC, NOVA and NWO-M grants; Target; the University of Padova, and the University Federico II (Naples).

We have made use of AsTROPY, a community-developed core PYTHON package for astronomy (Astropy Collaboration et al. 2013); APLPY, an open-source plotting package for Python (Robitaille \& Bressert 2012); the NASA/IPAC Extragalactic Database (NED), which is operated by the Jet Propulsion Laboratory, California Institute of Technology, under contract with the National Aeronautics and Space Administration; NASA's Astrophysics Data System Bibliographic Services; and the VizieR catalogue access tool operated at CDS, Strasbourg, France.

\section{REFERENCES}

Aditya J. N. H. S., Kanekar N., 2018, MNRAS, 481, 1578 Aditya J. N. H. S., Kanekar N., Kurapati S., 2016, MNRAS, 455, 4000 Allison J. R., Sadler E. M., Whiting M. T., 2012, PASA, 29, 221 Allison J. R., Sadler E. M., Meekin A. M., 2014, MNRAS, 440, 696 Allison J. R., et al., 2015, MNRAS, 453, 1249

Allison J. R., et al., 2016a, Astronomische Nachrichten, 337, 175 
Allison J. R., Zwaan M. A., Duchesne S. W., Curran S. J., 2016b, MNRAS, 462,1341

Allison J. R., et al., 2017, MNRAS, 465, 4450

Allison J. R., et al., 2019, MNRAS, 482, 2934

Astropy Collaboration et al., 2013, A\&A, 558, A33

Bahcall J. N., Ekers R. D., 1969, ApJ, 157, 1055

Baldry I. K., et al., 2014, MNRAS, 441, 2440

Becker R. H., White R. L., Helfand D. J., 1995, ApJ, 450, 559

Berg T. A. M., et al., 2017, MNRAS, 464, L56

Bigiel F., Leroy A., Walter F., Brinks E., de Blok W. J. G., Madore B., Thornley M. D., 2008, AJ, 136, 2846

Bird S., Garnett R., Ho S., 2017, MNRAS, 466, 2111

Bland-Hawthorn J., Maloney P. R., Stephens A., Zovaro A., Popping A., 2017, ApJ, 849, 51

Borthakur S., 2016, ApJ, 829, 128

Braun R., 2012, ApJ, 749, 87

Brookes M. H., Best P. N., Peacock J. A., Röttgering H. J. A., Dunlop J. S., 2008, MNRAS, 385, 1297

Bruzual G., Charlot S., 2003, MNRAS, 344, 1000

Carilli C. L., Walter F., 2013, AR\&A, 51, 105

Charlot S., Fall S. M., 2000, ApJ, 539, 718

Chippendale A. P., et al., 2015, in 2015 International Conference on Electromagnetics in Advanced Applications (ICEAA). IEEE, pp 541 - 544 (arXiv: 1509.00544)

Combes F., García-Burillo S., Braine J., Schinnerer E., Walter F., Colina L., 2013, A\&A, 550, A41

Condon J. J., Cotton W. D., Greisen E. W., Yin Q. F., Perley R. A., Taylor G. B., Broderick J. J., 1998, AJ, 115, 1693

Cooke R. J., Pettini M., Jorgenson R. A., 2015, ApJ, 800, 12

Cornwell T. J., Perley R. A., 1992, A\&A, 261, 353

Cornwell T. J., Golap K., Bhatnagar S., 2008, IEEE Journal of Selected Topics in Signal Processing, 2, 647

Crighton N. H. M., et al., 2015, MNRAS, 452, 217

Curran S. J., 2017, MNRAS, 470, 3159

Curran S. J., 2019, MNRAS, 484, 3911

Curran S. J., Whiting M. T., 2010, ApJ, 712, 303

Curran S. J., Whiting M. T., 2012, ApJ, 759, 117

Curran S. J., Murphy M. T., Pihlström Y. M., Webb J. K., Purcell C. R., 2005, MNRAS, 356, 1509

Curran S. J., Whiting M. T., Wiklind T., Webb J. K., Murphy M. T., Purcell C. R., 2008, MNRAS, 391, 765

Curran S. J., Allison J. R., Glowacki M., Whiting M. T., Sadler E. M., 2013, MNRAS, 431, 3408

Curran S. J., Reeves S. N., Allison J. R., Sadler E. M., 2016a, MNRAS, 459,4136

Curran S. J., Duchesne S. W., Divoli A., Allison J. R., 2016b, MNRAS, 462, 4197

Curran S. J., Hunstead R. W., Johnston H. M., Whiting M. T., Sadler E. M., Allison J. R., Athreya R., 2019, MNRAS, 484, 1182

Dale D. A., Helou G., Magdis G. E., Armus L., Díaz-Santos T., Shi Y., 2014, ApJ, 784, 83

Darling J., Giovanelli R., Haynes M. P., Bolatto A. D., Bower G. C., 2004, ApJ, 613, L101

Darling J., Macdonald E. P., Haynes M. P., Giovanelli R., 2011, ApJ, 742, 60

De Cia A., Ledoux C., Petitjean P., Savaglio S., 2018, A\&A, 611, A76

De Zotti G., Massardi M., Negrello M., Wall J., 2010, A\&AR, 18, 1

Decarli R., et al., 2016, ApJ, 833, 69

Decarli R., et al., 2019, submitted, preprint (arXiv:1903.09164), p. arXiv: 1903.09164

Dessauges-Zavadsky M., Ellison S. L., Murphy M. T., 2009, MNRAS, 396, L61

Dickey J. M., Mebold U., Marx M., Amy S., Haynes R. F., Wilson W., 1994, A\&A, 289, 357

Dickey J. M., Mebold U., Stanimirovic S., Staveley-Smith L., 2000, ApJ, 536, 756

Driver S. P., et al., 2018, MNRAS, 475, 2891
Dutta R., Srianand R., Gupta N., Momjian E., Noterdaeme P., Petitjean P., Rahmani H., 2017, MNRAS, 465, 588

Ellison S. L., Lopez S., 2009, MNRAS, 397, 467

Ellison S. L., Brown T., Catinella B., Cortese L., 2019, MNRAS, 482, 5694

Fernández X., et al., 2016, ApJ, 824, L1

Feroz F., Hobson M. P., 2008, MNRAS, 384, 449

Feroz F., Hobson M. P., Bridges M., 2009, MNRAS, 398, 1601

Geréb K., Maccagni F. M., Morganti R., Oosterloo T. A., 2015, A\&A, 575, A44

Giovanelli R., Haynes M. P., 2016, A\&AR, 24, 1

Giovanelli R., et al., 2005, AJ, 130, 2598

Glowacki M., et al., 2019, MNRAS, 489, 4926

Grasha K., Darling J., Bolatto A., Leroy A. K., Stocke J. T., 2019, ApJS, 245,3

Gupta N., et al., 2016, in Proceedings of MeerKAT Science: On the Pathway to the SKA. 25-27 May, 2016 Stellenbosch, South Africa (MeerKAT2016). p. 14 (arXiv: 1708.07371$)$

Gupta N., et al., 2018, MNRAS, 476, 2432

Heiles C., Troland T. H., 2003, ApJ, 586, 1067

Helfand D. J., White R. L., Becker R. H., 2015, ApJ, 801, 26

Hellwig H., Vessot R., Levine M., W. Zitzewitz P., W. Allan D., J. Glaze D., 1970, Instrumentation and Measurement, IEEE Transactions on, 19, 200

Hopkins A. M., Beacom J. F., 2006, ApJ, 651, 142

Hopkins A. M., et al., 2013, MNRAS, 430, 2047

Hurley-Walker N., et al., 2017, MNRAS, 464, 1146

Intema H. T., Jagannathan P., Mooley K. P., Frail D. A., 2017, A\&A, 598, A78

Isbell J. W., Xue R., Fu H., 2018, ApJL, 869, L37

Johnston S., et al., 2007, PASA, 24, 174

Jones M. G., Haynes M. P., Giovanelli R., Moorman C., 2018, MNRAS, 477,2

Kanekar N., Briggs F. H., 2004, New Astron. Rev., 48, 1259

Kanekar N., et al., 2014, MNRAS, 438, 2131

Kass R. E., Raftery A. E., 1995, Journal of the American Statistical Association, 90,773

Kauffmann G., Heckman T. M., 2009, MNRAS, 397, 135

Kauffmann G., et al., 2003, MNRAS, 346, 1055

Kauffmann G., et al., 2007, ApJS, 173, 357

Kennicutt Jr. R. C., 1998, ApJ, 498, 541

Kewley L. J., Geller M. J., Jansen R. A., 2004, AJ, 127, 2002

Kleiner D., et al., 2019, MNRAS, 488, 5352

Kuijken K., et al., 2019, A\&A, 625, A2

LaMassa S. M., Heckman T. M., Ptak A., Urry C. M., 2013, ApJL, 765, L33

Large M. I., Mills B. Y., Little A. G., Crawford D. F., Sutton J. M., 1981, MNRAS, 194, 693

Leahy D. A., et al., 2019, PASA, 36, e024

Liske J., et al., 2015, MNRAS, 452, 2087

Maccagni F. M., Morganti R., Oosterloo T. A., Geréb K., Maddox N., 2017, A\&A, 604, A43

Madau P., Dickinson M., 2014, ARA\&A, 52, 415

Magdis G. E., et al., 2014, ApJ, 796, 63

Martin A. M., Papastergis E., Giovanelli R., Haynes M. P., Springob C. M., Stierwalt S., 2010, ApJ, 723, 1359

Mauch T., Murphy T., Buttery H. J., Curran J., Hunstead R. W., Piestrzynski B., Robertson J. G., Sadler E. M., 2003, MNRAS, 342, 1117

McMullin J. P., Waters B., Schiebel D., Young W., Golap K., 2007, in Shaw R. A., Hill F., Bell D. J., eds, ASP Conf. Ser. Vol. 376, Astronomical Data Analysis Software and Systems XVI. Astron. Soc. Pac., San Francisco, p. 127

Meyer M., Robotham A., Obreschkow D., Westmeier T., Duffy A. R., Staveley-Smith L., 2017, Publ. Astron. Soc. Aust., 34

Morganti R., Oosterloo T., 2018, preprint, (arXiv:1807.01475)

Morganti R., Sadler E. M., Curran S., 2015, in Bourke T., Braun R., Fender R. P., et al. eds, Advancing Astrophysics with the Square Kilometre Array (AASKA14). Proc. Sci., p. 134 (arXiv: 1501.01091 )

Moss V. A., et al., 2017, MNRAS, 471, 2952 
Murray C. E., Stanimirović S., Goss W. M., Heiles C., Dickey J. M., Babler B., Kim C.-G., 2018, ApJS, 238, 14

Neeleman M., Prochaska J. X., Ribaudo J., Lehner N., Howk J. C., Rafelski M., Kanekar N., 2016, ApJ, 818, 113

Norris R. P., et al., 2011, Publ. Astron. Soc. Aust., 28, 215

Noterdaeme P., Petitjean P., Ledoux C., Srianand R., 2009, A\&A, 505, 1087

Noterdaeme P., et al., 2012, A\&A, 547, L1

Oosterloo T., Verheijen M. A. W., van Cappellen W., Bakker L., Heald G., Ivashina M., 2009, in Torchinsky S. A., van Ardenne A., van den BrinkHavinga T., van Es A. J. J., Faulkner A. J., eds, Wide Field Astronomy and Technology for the Square Kilometre Array. Proc. Sci., p. 70 (arXiv:0912.0093)

Péroux C., Deharveng J.-M., Le Brun V., Cristiani S., 2004, MNRAS, 352, 1291

Pihlström Y. M., Conway J. E., Vermeulen R. C., 2003, A\&A, 404, 871

Rafelski M., Wolfe A. M., Prochaska J. X., Neeleman M., Mendez A. J., 2012, ApJ, 755, 89

Rao S. M., Turnshek D. A., Nestor D. B., 2006, ApJ, 636, 610

Rao S. M., Turnshek D. A., Sardane G. M., Monier E. M., 2017, MNRAS, 471,3428

Reeves S. N., et al., 2016, MNRAS, 457, 2613

Reynolds J., 1994, AT Technical Document AT/39.3/040

Rhee J., Lah P., Briggs F. H., Chengalur J. N., Colless M., Willner S. P., Ashby M. L. N., Le Fèvre O., 2018, MNRAS, 473, 1879

Robitaille T., Bressert E., 2012, APLpy: Astronomical Plotting Library in Python, Astrophysics Source Code Library (ascl:1208.017)

Robotham A. S. G., Davies L. J. M., Driver S. P., Koushan S., Taranu D. S., Casura S., Liske J., 2018, MNRAS, 476, 3137

Robotham A. S. G., Bellstedt S., Lagos C. d. P., Thorne J. E., Davies L. J., Driver S. P., Bravo M., 2020, submitted, preprint (arXiv:2002.06980), p. arXiv:2002.06980

Roy N., Kanekar N., Chengalur J. N., 2013, MNRAS, 436, 2366

Sánchez-Ramírez R., et al., 2016, MNRAS, 456, 4488

Sault R. J., Teuben P. J., Wright M. C. H., 1995, in Shaw R. A., Payne H. E., Hayes J. J. E., eds, ASP Conf. Ser. Vol. 77, Astronomical Data Analysis Software and Systems IV. Astron. Soc. Pac., San Francisco, p. 433 (arXiv: astro-ph / 0612759)

Saunders W., et al., 2004, in Moorwood A. F. M., Iye M., eds, Society of Photo-Optical Instrumentation Engineers (SPIE) Conference Series Vol. 5492, Proc. SPIE. pp 389-400, doi: $10.1117 / 12.550871$

Schmidt M., 1959, ApJ, 129, 243

Serra P., Jurek R., Flöer L., 2012a, Publ. Astron. Soc. Aust., 29, 296

Serra P., et al., 2012b, MNRAS, 422, 1835

Serra P., et al., 2012c, MNRAS, 422, 1835

Shankar F., Weinberg D. H., Miralda-Escudé J., 2009, ApJ, 690, 20

Sharp R., et al., 2006, in Proc. SPIE. p. 62690G (arXiv: astro-ph/0606137), doi:10.1117/12.671022

Shimmins A. J., Bolton J. G., 1974, Australian Journal of Physics Astrophysical Supplement, 32, 1

Smith G. A., et al., 2004, in Moorwood A. F. M., Iye M., eds, Society of Photo-Optical Instrumentation Engineers (SPIE) Conference Series Vol. 5492, Proc. SPIE. pp 410-420, doi:10.1117/12.551013

Snellen I. A. G., Schilizzi R. T., Miley G. K., de Bruyn A. G., Bremer M. N., Röttgering H. J. A., 2000, MNRAS, 319, 445

Spergel D. N., et al., 2007, ApJS, 170, 377

Tacconi L. J., et al., 2013, ApJ, 768, 74

Ueda Y., Akiyama M., Ohta K., Miyaji T., 2003, ApJ, 598, 886

Villanueva V., et al., 2017, MNRAS, 470, 3775

Wang J., Koribalski B. S., Serra P., van der Hulst T., Roychowdhury S., Kamphuis P., Chengalur J. N., 2016, MNRAS, 460, 2143

Wolfe A. M., Gawiser E., Prochaska J. X., 2005, ARA\&A, 43, 861

Wright A. E., Griffith M. R., Hunt A. J., Troup E., Burke B. F., Ekers R. D., 1996, ApJS, 103, 145

Zafar T., Péroux C., Popping A., Milliard B., Deharveng J.-M., Frank S., 2013, A\&A, 556, A141

Zwaan M. A., van der Hulst J. M., Briggs F. H., Verheijen M. A. W., RyanWeber E. V., 2005, MNRAS, 364, 1467
This paper has been typeset from a $\mathrm{T}_{\mathrm{E}} \mathrm{X} / \mathrm{LT}_{\mathrm{E}} \mathrm{X}$ file prepared by the author. 